\title{
Quantum Spin Nematic States in Bose-Einstein Condensates
}

\author{
Fei Zhou \\ ITP, Utrecht University, Minnaert building, Leuvenlaan 4, 3584 CE Utrecht, The Netherlands
}

(October 25, 2018)

\begin{abstract}
We review some recent results on discrete symmetries and topological order in spinor BoseEinstein condensates (BECs) of ${ }^{23} \mathrm{Na}$. For spin one bosons with two-body scatterings dominated by a total spin equal to two channel, the BECs are in quantum spin nematic states at a low density limit. We study spin correlations in condensates at different limits and analyze hidden symmetries using a non-perturbative approach developed recently. We further more investigate the influence of hidden $Z_{2}$ symmetries and $U(1)$ quantum orders in symmetry partially retored states, particularly the effects on topological excitations.
\end{abstract}

\section{INTRODUCTION}

BECs of alkali atoms first created and studied experimentally in 1995-1997 revived theorists' interest on this subject [1]:2.3]. Because the Zeemann coupling in general provides stronger confining potentials for atoms compared with other trapping methods, and a rf evaporative cooling is needed to achieve BECs, the first generation of BECs was realized in magnetic traps in laboratories. In those early experiments, spins of atoms were completely polarized and BECs with single component were achieved, similar to the BEC of ${ }^{4} \mathrm{He}$ studied a few decades ago. Apart from the complication of the confining potentials, the theoretical description of BECs of alkali atoms with positive scattering lengths in magnetic traps can be carried out in the Gross-Pitaveskii approach

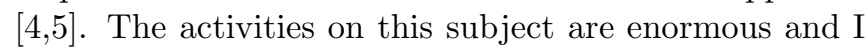
should refer to an excellent review by Dalfovo et al. [6].

The physical properties of single component BECs of alkali atoms depend on two length scales. The interatomic distance, which is about a few hundred nanometers in most of experiments, characterizes the kinetic energy in the problem. The BEC transition temperature which is determined by this length scale is about a few micro Kelvins or lower in experiments. The upper limit of the atomic density in BECs and the transition temperature are imposed by three-body recombination processes which lead to trap losses in experiments [7]. The second length scale is the two-body scattering length, which is about a few nanometers for alkali atoms. It characterizes the interactions between atoms and therefore the chemical potentials. Given the density of atoms and the scattering lengths in most of experiments, the chemical potentials are typically of the order of a few hundreds nano Kelvins. Amazingly, the scattering length depends on magnetic fields and the variation in scattering lengths is particularly substantial when the atoms are close to the Feshbach resonance [8,9]. This remarkable property of alkali atoms was observed in $N a$ by Inouye et al. [10], in $R b$ by Courteille et al. [11], Roberts et al. [12] and recently Cornish et al. [14], in $C s$ by Vuletic et al. [13]. Collapsing of BECs of ${ }^{7} \mathrm{Li}$ atoms with negative scatter- ing lengths was studied by Sackett et al. 15]. Dynamics of collapsing was investigated theoretically in [16] and remains to be fully understood.

Correspondingly, there are at least two classes phenomena investigated very extensively by experimentalists. The first one is connected with quantum coherence of BECs. Quantum interference between two condensates (both spatial and temporal) and the creation of vortices belong to this category $17,18,19,20,21$. For instance, vortices were first created using a combination of internal transition and external rotation by Matthews [19], as theoretically suggested by Williams and Holland in 1999. Arrays of vortices were found by Madison et al. [20], who stirred BECs by rotating the trap. Superfluidity of BECs has been examined by Raman et al. [22] who demonstrated frictionless flow and by Marago et al. [23], who observed beautiful undamped scissors mode suggested by Guery-Odelin and Stringari in 1999 [24].

The second class is related with scatterings between atoms, or the collective behavior of BECs. The AC Josephson effect of two internal states with different scattering lengths and zero sound excitations at coherent limit fall into this category [25,26, 27,28]. The second class phenomena are of particular interest from condensed matter physics points of view and let me describe two interesting experiments in some details. In the experiment in [25], Hall et al. first prepared ${ }^{87} R b$ in a hyperfine spin state $\mid F=1, m_{F}=-1>$ in magnetic traps. By applying external microwaves or so called $\frac{\pi}{2}-$ pulses, they successfully transformed about fifty percent of atoms from $|1,-1\rangle$ state into $\mid 2,-2>$ state. The chemical potentials of these two condensates are different because of different scattering lengths. After certain waiting time $T$, a fraction of the condensate at $|2,-2\rangle$ state is transformed back to $|1,-1\rangle$ state by another $\frac{\pi}{2}$ pulse. The interference between the original condensate and the newly converted condensate depends on the accumulated phases of two condensates after time $T$, which are different because of two different chemical potentials $\mu_{i}=4 \pi a_{i} \rho / M$ for condensates with a density $\rho$, a scattering length $a_{i}$ and an atomic mass $M$. The temporal dependence of the interference observed by Hall et al. does exhibit periodical oscillations at $m s$ scale, expected 
by a simple estimate of the chemical potentials.

The observation of zero sound excitations in ${ }^{23} \mathrm{Na}$ BECs was carried out by Stamper-Kurn et al. [26] in 1998. After a temporal magnetic field was applied to disturb the BEC, a breathing of condensate was observed at a temperature much lower than the BEC transition temperature. Snap shots taken at $5 \mathrm{~ms}$ apart indicate oscillations in the size of the BEC with a period of a few $m s$, corresponding to the time interval for a sound like excitation to travel from one end of the BEC to the other one. The sound velocity, being proportional to the square root of the compressibility, depends on the scattering lengths and appears to be consistent with the frequency of the oscillations. These oscillations were also shown quickly damped at the temperature close to the transition point, caused by scatterings with a thermal cloud.

BECs with multiple components were created and studied a few years later 29, 30,31. Particularly, Stamper-Kurn et al. successfully used optical beams to trap ${ }^{23} \mathrm{Na}$, which confines alkali atoms independent of spins and liberates spin degrees of freedom [30]. For alkali atoms ${ }^{23} \mathrm{Na}$ or ${ }^{87} \mathrm{Rb}$, with nuclear spin $I=3 / 2$ and electrons at $s$ orbits, the energy splitting between hyperfine multiplets is of order $100 \mathrm{mk}$. At temperatures as low as a few hundred nano Kelvin, ${ }^{23} \mathrm{Na}$ and ${ }^{87} \mathrm{Rb}$ atoms can be considered as simple bosons with a hyperfine spin $F=1$. Liberation of spins in BECs in an optical environment indeed leads to a new dimension of studying BECs, especially spin correlated condensates. This further inspired many theoretical activities on spinor Bose-Einstein condensates 32,33,34]. The spin correlated states in ${ }^{23} \mathrm{Na}$ were afterwards studied experimentally by Stenger et al. [31.

The new complication in BECs in optical traps surely arises from the extra internal degree of hyperfine spins. When $N_{m_{F}}\left(m_{F}=0, \pm 1\right)$ atoms condense at each of the hyperfine states $\mid F, m_{F}>$ but all condense at the lowest orbital state, the ground state of $N\left(=\sum_{m_{F}} N_{m_{F}}\right)$ noninteracting spin $F=1$ bosons has a $\mathcal{D}_{s}$-fold spin degeneracy,

$$
\mathcal{D}_{s}=\frac{(N+2)(N+1)}{2},
$$

by contrast to its magnetically trapped cousins. Presumably, hyperfine spin-dependent scatterings lift the degeneracy and lead to spin correlated states. Optically trapped BECs therefore set up a platform for the study of the quantum magnetism in many boson systems and add a new twist to already extremely fascinating systems.

For ultracold atoms in BECs, the spin-dependent two -body interaction is characterized by $U_{2}\left(\mathbf{r}_{1}, \mathbf{r}_{2}\right)=\delta\left(\mathbf{r}_{1}-\right.$ $\left.\mathbf{r}_{2}\right)\left[c_{0}+c_{2} \mathbf{F}_{\mathbf{1}} \dot{\mathbf{F}}_{\mathbf{2}}\right]$, as suggested in an early paper [32]. Here $c_{0}=\left(g_{0}+2 g_{2}\right) / 3, c_{2}=\left(g_{2}-g_{0}\right) / 3 ; g_{F}=4 \pi \hbar^{2} a_{F} / M, \mathrm{M}$ is the mass of the atom and $a_{F}$ is the s-wave scattering length in the total spin $F$ channel. Thus, spin correlation in BECs is determined by $c_{2}$. For ${ }^{87} R b$ studied experimentally by the JILA group [29], $g_{2}<g_{0}$ or $c_{2}<0$ and the scattering is dominated by the total spin $F=0$ channel. In the ground state, all spins of atoms prefer to align in a certain direction and have a maxima magnetization 32 . For ${ }^{23} \mathrm{Na}$ studied experimentally by the MIT group [30], the scattering between ${ }^{23} \mathrm{Na}$ atoms is dominated by the total spin $F=2$ channel, i.e. $g_{2}>g_{0}$. The scatterings between ${ }^{23} \mathrm{Na}$ atoms thus lead to "antiferromagnetic" spin correlation. In this article, I will fully explore the properties of the BEC of ${ }^{23} \mathrm{Na}$.

Efforts have been made to understand the ground state properties, exact excitation spectra, collective modes and topological excitations. Theoretically, issues of spin ordering in the spinor BECs are of particular interest and were treated by Ho, and also Ohmi and Machinda in 1998. In both works, the spin ordering was discussed in a mean field approximation, in the context of the GrossPitaveskii equation. Ho also obtained two branches spin wave excitations by a Gaussian expansion.

The first attempt to understand spin correlation beyond the mean field limit was made by Law, $\mathrm{Pu}$ and Bigelow [34]. Law et al. observed that the microscopic Hamiltonian of $\mathrm{N}$ spin-1 Bosons interacting via a spin-dependent two-body potential given above commutes with the total spin of the system; the spin is a good quantum number and the exact eigenstates should be eigenstates of the total spin operator. When $c_{2}>0$, the ground state should be a spin singlet and rotationally invariant. This was explicitly demonstrated in a zero mode approximation, where the finite momentum sector of the Hilbert space was neglected [34]. Using a four-wave mixing method, Law et al. obtained the exact excitation spectrum in a $0 D$ limit, a limit where all spatial fluctuations are neglected. These results in $0 D$ case were also derived by Castin and Herzog [35], using a geometrical description very close to the one I am going to introduce in this paper. Law et al.' $\mathrm{s}$ results, though are barely relevant to the particular experiment done by Stenger et al., given the number of atoms, time scales during which the experiment was performed and small biased magnetic fields in the experiment, nevertheless inexplicitly reveal the role of symmetry restoring processes and the nature of quantum fluctuations of the spin order in the problem. The even-odd effect in the excitation spectrum observed by Law et al. further implies a discrete symmetry in the problem which I believe has a profound origin.

Ho and Yip later found that the singlet states obtained by Law et al. are rather sensitive to an external magnetic field 36]. In a thermal dynamical limit, an infinitesimal magnetic field will stablize the symmetry broken state and the rotationally invariant state is irrelevant as far as experiments with a small magnetic field are concerned. This feature of ${ }^{23} \mathrm{Na}$ was later realized to relate with the level spacing of the low lying excitations 37.38. For the $0 D$ dimensional BEC, the energy of low 
lying excitations scales down as the number of atoms and the quasi-degeneracy makes the system ultrasensitive to magnetic fields. A symmetry broken state, which can be constructed out of low lying excitations has an energy inversely proportional to the number of atoms involved and can be pinned by an infinitesimal field when the number of atoms is infinity.

Topological excitations in the spinor BECs of ${ }^{23} \mathrm{Na}$ have been paid a special tribute to by a few groups. Linear defects were studied by Leonhart and Volovik [39], who pointed out the existence of Alice strings in the BECs of ${ }^{23} \mathrm{Na}$. Textures of the Skyrmion type were pointed out in [32]; and most recently, point defects were examined [40]. Except the work of Leonhart and Volovik, most of the works on this subject were based on Ho's original identification of the internal space, which missed the important discrete symmetry of $Z_{2}$ type. Therefore, many new aspects of topological defects studied in this paper have never been investigated. For instance, in a recent work 40], the authors applied the nonlinear $\sigma$ model derived in [37]38] to study static and dynamical properties of hedgehogs. Unfortunately, the authors of that work failed to observe the correct internal space. Many aspects of the point defects in the BECs of ${ }^{23} \mathrm{Na}$, particularly those under the influence of the entanglement of the phase and spin orders including the homotopical indistinguishability between \pm hedgehogs, between hedgehogs and closed loop $\pi$-disclinations were previously discussed [37]. I will review those results in this article.

The pursue of having a nonperturbative approach beyond a zero dimension approximation was first made in [37,38]. That approach allows one to identify that the low energy spin dynamics in the BECs of spin one atoms with antiferromagnetic interactions belongs to the same universality class of the nonlinear- $\sigma$ model(NL $\sigma \mathrm{M})$, a relatively well known subject, thanks to many studies in the context of field theory. The NL $\sigma \mathrm{M}$ also became known to many condensed matter physicists working with antiferromagnetic systems after a paper in 1983 [41.

In 37,38, the spin dynamics in the problem of $\mathrm{N}$ spin one bosons interacting via a spin-dependent two-body potential with $c_{2}>0$ was mapped into an $o(n)$ nonlinear sigma model (NL $\sigma M) . n=3$ at the zero magnetic field limit and $n=2$ in the presence of a weak magnetic field. With the help of this mapping, the many-body aspect of ${ }^{23} \mathrm{Na}$ becomes rather explicit and many properties including spin correlations in $1 d$ can be studied. Furthermore, energetics and dynamics of topological excitations in BECs can therefore be easily analyzed in this approach.

At a low density limit, it is also found that the ground state has a similar discrete symmetry as that of a uniaxial nematic phase of liquid crystals, except a $\pi$-phase factor as discussed extensively in 37,38 and for this reason the condensate is at a quantum spin nematic state $(Q S N S)$. The ground state degeneracy space is $\left[U(1) \times S^{n}\right] / Z_{2}$, with $n$ depending on a magnetic field. The antiferromagnetic scatterings between cold atoms lead to a $Z_{2}$ symmetry breaking.

This discrete symmetry was later appreciated by Demler et al. in the context of low lying excitations [42,43]. Demler et al. noticed that the $Z_{2}$ symmetry in a symmetry broken state actually also appears in all low lying states. By imposing a proper projection in a functional integral approach, they showed that the full low energy physics in the phase and spin sector is given by $Z_{2}$ gauge fields coupled with two matter fields characterized by an $X Y$ model and a NL $\sigma \mathrm{M}$. This work as well as the previous work 37,38 open a new door for the studies of many-boson states. Many issues, such as fractionalization of topological excitations, fractionalization of quasiparticles and topological order were studied and investigated with the help of this mapping [44,45. A number of new predictions have been made for the spinor BEC, most of which have never been studied experimentally. The mapping also provides a starting point for the future study of some strongly correlated fermionic systems.

The purpose of this article is to provide a guideline and introduction to some ideas on the spinor BECs developed very recently in [37,38. Through out the article, I will emphasis on the concepts of topology, at levels of both the mean field approximation, which is suitable for a discussion on symmetry broken states and beyond, which is essential for the study of symmetry restored states. This point of view might be dramatically different from the conventional ones shared by atomic physicists. On the other hand, it seems to me that the nature has chosen to behave in such a unconventional, complicated way that I am left with no alternatives, or a simpler presentation. It also appears to my collaborators and me during this investigation that this is a subject full of surprises and new issues keep on poping up. At the time of writing, I present results which I believe to be relevant to BECs investigated in experiments. There are many other issues still remaining to be explored. Furthermore, some descriptions given here are qualitative and conceptional, so as to be communicated in a most efficient way. Like many other new subjects, a more quantitative aspect of some properties can be achieved only after basic notions are established and will be present in a series of separate works. At last, I should point out that many strongly correlated electronic systems are believed to share very similar topological properties [46,47] and therefore the BECs might be systems where some of basic ideas established in strongly correlated electron systems can be tested experimentally. Understanding of the spinor BEC might as well shed some light on other Fermionic superfluids such as p-wave triplet superconductors.

So, I will review the nonperturbative approach recently formulated. Results obtained previously by different groups with other methods can be reproduced in 
this new representation. I will of course go beyond previous framework and outline many novel results obtained in the last two years $37,38,42,43,44,45$. In section II, a mapping from the original microscopic model of $\mathrm{N}$ spin one Bosons with $c_{2}>0$ into a $N L \sigma M$ at long wave length limit will be introduced. Furthermore, the corresponding $\mathrm{N}$-body wave function is shown to be invariant under a $180^{\circ}$ rotation of a vector $\mathbf{n}$ and a $U(1)$ gauge transformation. At last, $Z_{2}$ gauge fields are introduced in the model to enforce the discrete symmetry.

In section III, symmetry broken states are examined. The internal order parameter space for the BEC is identified as $\left[S^{1} \times S^{2}\right] / Z_{2}$ (zero field limit). Linear, point defects and "particle like" textures are discussed with an emphasis on the influence of the $S^{2}$ group and the discrete $Z_{2}$ symmetry. Particularly, a family of strings representing $\pi$ spin disclinations superimposed by superfluid vortices of half integer circulation are studied. The distinguishabilities of \pm hedgehogs, both physical and homotopical, are analysised. The connection between closed loop $\pi$ disclinations and hedgehogs is established.

The effect of an external magnetic field is also reviewed. The NL $\sigma \mathrm{M}$ in this case has two components instead of three at zero external field. The ground state is degenerate in a manifold $\left[U(1) \times S^{1}\right] / Z_{2}$.

In section IV, symmetry unbroken states are studied in the scheme of the NL $\sigma \mathrm{M}$. The possibility of having nematic order-disorder phase transitions and the nature of spin correlation are addressed. The ground state of $\mathrm{N}$ spin one Bosons interacting via $c_{2}>0$ spin-dependent two-body potentials in $1 \mathrm{~d}$ limit is also present. In section $\mathrm{V}$, the influence of the $Z_{2}$ symmetry on the symmetry unbroken states is discussed. One of the most interesting consequencies of having $Z_{2}$ symmetries is the possible fractionalization of topological excitations. Under the influence of the $Z_{2}$ symmetry, it is shown that vortices can be fractionalized into half-vortices. It is also pointed out that instead of a condensate of individual atoms, one can have a singlet pair condensate, which supports free $Z_{2}$ vortices.

In section VI, the property of connection fields is analysised and hidden topological order is identified in spin disordered BECs. The existence "topological order" is argued due to an "order from disorder" mechanism.

In section VII, I discuss the symmetry restoring of a finite size nematically ordered state. Quantum mechanical nature of the order parameters in finite size ${ }^{23} \mathrm{Na}$ BEC was addressed in connections of experimental probes, taking into account the metastability of the atomic gas. In section VIII, I make some remarks on the relation between the BECs considered here and other strongly correlated magnetic systems.

\section{GEOMETRIC DESCRIPTION}

The inconsistency between the mean field point of view taken in 32, 33] and the spin singlet ground state obtained in 34 , lies in the heart of the quantum symmetry restoring. If one starts with the symmetry broken solution in the GPE approach, for instance, one can ask how the rotation symmetry is restored after all and when that happens. A more practical question would be, given the life time of the metastable BEC gaseous cloud studied in the experiment, is the rotationally invariant state relevant? This question motivates us to develop a nonperturbative approach. The answer to this question requires a complete characterization of nonlinear spin dynamics.

The second issue concerning us is the validity of the zero mode approximation 34. Though the exact solutions in a zero mode approximation provide information of low lying excitations, they fail to yield spatial-temporal spin fluctuations, which are essential for the most general description of the ground state of $N$ interacting $F=1$ Bosons. The finite momentum sector of Hilbert space is clearly critical for such a discussion.

It is also worth commenting that the GPE approach with three components 32,33], on the other hand, does permit a gradient expansion around the symmetry broken solution in principal. However, such a procedure is feasible only when the derivation of the true ground state from the uniform solution is small. Practically, the GPE approach works in a small vicinity of the symmetry broken solution in the order parameter space and is far from being a general description.

The third and perhaps the most important one is the description of nonlinear spin dynamics in the spinor BEC. The failure of the generalized GPE approach far away from its mean field solution is largely due to the fact that the collective variables chosen there are not suitable for a description of the nonlinear spin dynamics. For this reason, in the framework of the GPE approach, the interactions between low lying excitations(spin waves) which eventually disrupt the symmetry broken state are barely tractable.

For instance, when the zero point rotation energy of each atom caused by two-body scatterings is high enough, long-range spin order predicted in the GPE approach is destroyed even at zero temperature(see details in section IV). In that case, spin correlations can be nontrivial and yet not characterized by the simple solutions of the GPE. In addition, the spin correlated state in a very anisotropic trap, or $1 d$, is clearly beyond the validity of the GPE approach.

Efforts along this line had been carried out recently. To have a general approach of studying spin correlated states of spin one bosons with $c_{2}>0$ was the purpose of those works. It is reviewed in the rest of this section. I will demonstrate a geometrical approach which allows 
us to go far beyond the three-component Gross-Pitaveskii approach and explore a variety of possible spin correlated states.

\section{A. NL $\sigma \mathrm{M}$ characterization of spin dynamics}

Following the discussions above, to study the spin correlated $\mathrm{BEC}$, it is important that a set of collective coordinates are correctly defined. Idealistically, these variables should be chosen to a) completely characterize the nonlinear spin dynamics in the whole parameter space and b) to form a simplest representation for two-body spin dependent scatterings. For this purpose, we use two vectors $\left(\boldsymbol{\Omega}_{1}, \boldsymbol{\Omega}_{2}\right)$ living on two unit spheres to describe the nonlinear spin dynamics in the presence of spin-dependent scatterings. We are going to map the $\mathrm{N}$ interacting spin one Boson problem into an $O(3) \mathrm{NL} \sigma \mathrm{M}$ self-consistently.

We make two assumptions to proceed further. First, there exists a class of spatially varying $\mathrm{N}$-body wave functions, out of which the $\mathrm{N}$ interacting spin-1 Bosons ground state and the low lying excitations can be constructed. The whole class is characterized by a few collective coordinates ( $\boldsymbol{\Omega}_{1,2}$ introduced below).

Second, the energy of this class N-body wave functions under consideration can be expressed in terms of a finite number of collective variables. Quantization of the collective modes yields elementary low lying excitations, which can interact with each other strongly. The exact N-body wave function of the ground state is characterized by the zero point motions of collective variables and their multicouplings. Practically, in most limits, the ground state is conveniently characterized by the correlation functions. The low energy spin excitations are of a collective nature; the excitations of individual spins are neglected in the leading order approximation $O(1 / N)$.

The correctness of this approach is justified by the following measure: The resultant theory which is written in terms of collective variables instead of N-body wave functions of individual atoms should produce the correct nonlinear spin dynamics, such as Euler's equation of motion, conservation laws, at the long wave length limit. The theory is self-consistent if this measure is satisfied. The derivation of the mapping was obtained and summarized in 37,38.

To describe the spin correlated BEC, it is most convenient to introduce Weyl representation of $S U(2)$ involving polynomials of a unit vector $(u, v)$ 48,49. Each unit vector is represented by a point $\Omega$ on a sphere with polar coordinates $(\theta, \phi)$; namely

$$
u=\exp \left(i \frac{\phi}{2}\right) \cos \frac{\theta}{2}, v=\exp \left(-i \frac{\phi}{2}\right) \sin \frac{\theta}{2} \text {. }
$$

The corresponding hyperfine spin operators are

$$
\begin{gathered}
F^{+}=u \frac{\partial}{\partial v}, F^{-}=v \frac{\partial}{\partial u} \\
F_{z}=\frac{1}{2}\left(u \frac{\partial}{\partial u}-v \frac{\partial}{\partial v}\right) .
\end{gathered}
$$

The scalar product between two wave functions $g$ and $f$ is defined as $\int g^{*}(u, v) f(u, v) d \Omega / 4 \pi$. (We reserve $\boldsymbol{\Omega}$ for the spin rotation discussed below.)

Under spin rotations $\mathcal{R}=\exp \left(i F_{z} \chi_{1} / 2\right) \exp \left(i F_{y} \theta_{1} / 2\right)$ $\exp \left(i F_{z} \phi_{1} / 2\right), u$ and $v$ transform into

$$
\begin{aligned}
& u\left(\boldsymbol{\Omega}_{\mathbf{1}}, \chi_{1}\right)= \\
& \exp \left(i \frac{\chi_{1}}{2}\right)\left(\cos \frac{\theta_{1}}{2} \exp \left(-i \frac{\phi_{1}}{2}\right) u+\sin \frac{\theta_{1}}{2} \exp \left(i \frac{\phi_{1}}{2}\right) v\right) \\
& v\left(\boldsymbol{\Omega}_{\mathbf{2}}, \chi_{2}\right)= \\
& \exp \left(-i \frac{\chi_{2}}{2}\right)\left(-\sin \frac{\theta_{2}}{2} \exp \left(-i \frac{\phi_{2}}{2}\right) u+\cos \frac{\theta_{2}}{2} \exp \left(i \frac{\phi_{2}}{2}\right) v\right)
\end{aligned}
$$

where $\boldsymbol{\Omega}_{1,2}=\left(\theta_{1,2}, \phi_{1,2}\right)$. The following identities hold

$$
\begin{aligned}
& \int u^{*}\left(\boldsymbol{\Omega}_{1}\right) \mathbf{F} u\left(\boldsymbol{\Omega}_{1}\right) \frac{d \Omega}{4 \pi}=\frac{1}{6} \boldsymbol{\Omega}_{1}, \\
& \int v^{*}\left(\boldsymbol{\Omega}_{1}\right) \mathbf{F} v\left(\boldsymbol{\Omega}_{1}\right) \frac{d \Omega}{4 \pi}=-\frac{1}{6} \boldsymbol{\Omega}_{1} .
\end{aligned}
$$

Spin one wave functions are polynomials of degree two in $u$ and $v$. $\sqrt{3} u^{2}, \sqrt{6} u v, \sqrt{3} v^{2}$ correspond to $m=1,0,-1$ states. All $F=1$ states can also be expressed in term of $\sqrt{6} u\left(\boldsymbol{\Omega}_{\mathbf{1}}\right) v\left(\boldsymbol{\Omega}_{\mathbf{2}}\right)$ with $\boldsymbol{\Omega}_{1,2}$ properly chosen. The examples are given in Fig.1. The comparison between the polynomial representation and the usual vector representation is given in the Appendix A.

The Hamiltonian for spin one bosons can be written as

$$
\begin{aligned}
& \mathcal{H}=-\frac{1}{2 M} \sum_{\alpha} \nabla_{\alpha}^{2}+\sum_{\alpha, \beta}\left[\frac{c_{0}}{2}+\frac{c_{2}}{2} \mathbf{F}_{\alpha} \cdot \mathbf{F}_{\beta}\right] \delta\left(\mathbf{r}-\mathbf{r}^{\prime}\right) \\
& +\sum_{\alpha} \mathbf{F}_{z \alpha} g \mu_{B} H .
\end{aligned}
$$

The second term is the hyperfine spin-dependent interaction and the last term is the coupling with an external magnetic field $\mathbf{H}=H \mathbf{e}_{z} ; g$ is the g-factor of bosons and $\mu_{B}$ is the Bohr magneton.

The wave function for $\mathrm{N}$ spin one Bosons can generally be written as

$\Psi\left(\left\{\mathbf{r}_{\alpha}\right\}\right)=\mathcal{S} \Pi_{\alpha=1 \ldots N} \Phi_{N_{\alpha}}\left(\mathbf{r}_{\alpha}\right) \sqrt{6} u_{\alpha}\left(\boldsymbol{\Omega}_{1 \alpha}\left(\mathbf{r}_{\alpha}\right)\right) v_{\alpha}\left(\boldsymbol{\Omega}_{2 \alpha}\left(\mathbf{r}_{\alpha}\right)\right)$.

$\mathcal{S}$ is to symmetrize the wave function; $N_{\alpha}$ labels oneparticle states. The phase $\left[\chi_{1}-\chi_{2}\right] / 2$ corresponds to a gauge transformation in the complex field $\Phi_{N_{\alpha}}$ introduced above. Without losing generalities, we set $\chi_{1}=\chi_{2}=0$. 
By taking $\boldsymbol{\Omega}_{1 \alpha, 2 \alpha}=\boldsymbol{\Omega}_{1,2}(\mathbf{r})$ and $\Phi_{N_{\alpha}}(\mathbf{r})=\Phi(\mathbf{r}) / \sqrt{N}$ $\left(\Phi(\mathbf{r})\right.$ is a complex scalar field), one chooses $\boldsymbol{\Omega}_{1,2}, \Phi(\mathbf{r})$ as the collective variables of the $\mathrm{N}$ interacting spin one Bosons. One obtains a Hamiltonian for the spin and superfluid sectors

$$
\begin{aligned}
& \mathcal{H}=\mathcal{H}_{s}+\mathcal{H}_{c}+\mathcal{H}_{e x}, \\
& \mathcal{H}_{s}=\frac{1}{2} \int d \mathbf{r}\left[\frac{1}{M}(\nabla \mathbf{n}(\mathbf{r}))^{2} \Phi^{*} \Phi+4 c_{2} \mathbf{L}^{2}(\mathbf{r})\left|\Phi^{*} \Phi\right|^{2}\right], \\
& \mathcal{H}_{c}=\frac{1}{2} \int d \mathbf{r}\left[\frac{1}{M}|\nabla \Phi(\mathbf{r})|^{2}+4 c_{0}\left|\Phi^{*}(\mathbf{r}) \Phi(\mathbf{r})\right|^{2}\right], \\
& \mathcal{H}_{e x}=g \mu_{B} H \int d \mathbf{r} \Phi^{*}(\mathbf{r}) \Phi(\mathbf{r}) \mathbf{e}_{z} \cdot \mathbf{L}(\mathbf{r}),
\end{aligned}
$$

Here $\mathbf{n}(\mathbf{r})=\left(\boldsymbol{\Omega}_{1}+\boldsymbol{\Omega}_{2}\right) / 2, \mathbf{L}(\mathbf{r})=\left(\boldsymbol{\Omega}_{1}-\boldsymbol{\Omega}_{2}\right) / 2$. Subscripts $s, c$ and ex label spin, charge and external fields. In the spin sector, the first term is the energy cost of twisting $\mathbf{n}$ in space, representing the spin stiffness; the second term is the effective "rotation" energy in the presence of a finite spin moment $\mathbf{L}$.

A local spin-phase coupling term, $\mathcal{H}_{s c}$, which represents the superflow in the BECs due to the Berry's phases under spin rotation, does exist 37. This term, however, is linear in terms of $\mathbf{L}$ and quadratic in terms of spatial gradient and is much smaller than $\mathcal{H}_{s}, \mathcal{H}_{c}$ at the long wave length limit. Moreover, $\mathcal{H}_{s c}$ vanishes in a state where $\mathbf{L}$ is zero. Based on these observations, it was pointed out that the local coupling is negligible 38 .

In the most interesting limit, we can introduce $\Phi(\mathbf{r})=$ $\sqrt{\rho(\mathbf{r})} \exp (i \chi(\mathbf{r}))$; the local spin density is $\mathbf{l}(\mathbf{r})=$ $\mathbf{L}(\mathbf{r}) \rho(\mathbf{r})$. $\mathbf{n}$ and $\mathbf{l}$ satisfy the constraint

$$
\mathbf{n}(\mathbf{r}) \cdot \mathbf{l}(\mathbf{r})=0 .
$$

$\rho$ and $\phi, \mathbf{n}(\mathbf{r})$ and $\mathbf{l}(\mathbf{r})$ obey the following commutation relations,

$$
\begin{aligned}
& {\left[\rho(\mathbf{r}), \chi\left(\mathbf{r}^{\prime}\right)\right]=i \hbar \delta\left(\mathbf{r}-\mathbf{r}^{\prime}\right) ;} \\
& {\left[\mathbf{n}_{\alpha}(\mathbf{r}), \mathbf{n}_{\beta}\left(\mathbf{r}^{\prime}\right)\right]=0,} \\
& {\left[\mathbf{l}_{\alpha}(\mathbf{r}), \mathbf{n}_{\beta}\left(\mathbf{r}^{\prime}\right)\right]=i \hbar \epsilon^{\alpha \beta \gamma} \mathbf{n}_{\gamma} \delta\left(\mathbf{r}-\mathbf{r}^{\prime}\right),} \\
& {\left[\mathbf{l}_{\alpha}(\mathbf{r}), \mathbf{l}_{\beta}\left(\mathbf{r}^{\prime}\right)\right]=i \hbar \epsilon^{\alpha \beta \gamma} \mathbf{l}_{\gamma} \delta\left(\mathbf{r}-\mathbf{r}^{\prime}\right) .}
\end{aligned}
$$

$\epsilon^{\alpha \beta \gamma}$ is an antisymmetric tensor. The second identity in Eq.10 is valid only when $\mathbf{L}$ per atom is much less than unity and $\mathbf{n}(\mathbf{r})$ can be considered as a classical "vector".

The equation of motion for the superfluid and the spin sector can be derived as

$$
\begin{aligned}
& \partial_{t} \chi=4 c_{0} \rho-\frac{1}{M \rho} \nabla^{2} \rho+\frac{1}{2 M}(\nabla \rho)^{2} J, \\
& \partial_{t} \rho=\nabla \cdot\left(\frac{\rho}{M} \nabla \chi\right), \\
& \partial_{t} \mathbf{n}=4 c_{2} \mathbf{n} \times\left(\mathbf{l}-\frac{g \mu_{B} \mathbf{H}}{4 c_{2}}\right), \\
& \partial_{t} \mathbf{l}=-\frac{\rho(\mathbf{r})}{M} \mathbf{n} \times \nabla^{2} \mathbf{n}
\end{aligned}
$$

where the low frequency and long wavelength limits have been taken. $J=\left[\chi, \rho^{-1}\right] / i \hbar$. Eq.11 determines the nonlinear spin dynamics in the BECs in the long wave length limit.

The first two equations indicate the finite compressibility or chemical potential in the presence of scatterings between atoms, and the usual current conservation law. The first equation suggests the AC Josephson effects involving two condensates in symmetry broken states. It also implies the noncommutative nature of the phase degree freedom $\chi$ and the Hamiltonian $\mathcal{H}_{c}$ for interacting atoms. This leads to the quantum fluctuations of the condensate phase $\chi$ and symmetry retoring in a finite condensate. Linearizing these equations yields excitations of the nature of zeroth sounds, which involve a periodical compression of the density and a periodical modulation of the phase of the condensation. The sound velocity is $v_{c}=\sqrt{4 c_{0} \rho / M}$.

The third equation shows that $\mathbf{n}$ precesses in the presence of the spin density $\mathbf{l}(\mathbf{r})$ due to the two-body hyperfine spin dependent scatterings. Again, it results from the noncommutative nature of the spin order $\mathbf{n}$ and $\mathcal{H}_{s}$. The profound phenomenon of the quantum symmetry restoring of $\mathbf{n}$ in a finite size spinor BEC is driven by this property. In homogeneous BECs, the fourth equation can be considered as the "conservation law" for the spin density $\mathbf{l}(\mathbf{r})$; the corresponding spin current density is $\mathbf{j}_{\alpha}=\rho M^{-1} \epsilon_{\alpha \beta \eta} \mathbf{n}_{\eta} \nabla \mathbf{n}_{\beta} . \quad \rho$ is the density of atoms. For the sake of simplicity, we will assume the system is homogeneous from now on.

When $c_{2}>0$, the "rotation" energy in $\mathcal{H}_{s}$ is positive. At the zero field limit, there exists a saddle point solution of Eq.8 for the spin sector

$$
\mathbf{n}(\mathbf{r})=\mathbf{n}_{0}, \mathbf{l}(\mathbf{r})=0 .
$$

$\mathbf{n}_{0}$ lies on the unit sphere. The solution is schematically shown in Fig.1.

$u\left(\mathbf{n}_{0}\right) v\left(\mathbf{n}_{0}\right)$ represents state $(0,1,0)^{T}$ with the quantization axis pointing along $\mathbf{n}_{0}$. It is easy to confirm that this solution at $H=0$ corresponds to the "polar" state found in Ref. [32]. By expanding Eq.11 around the mean field solution, we obtain the spin waves with a sound like spectrum $\omega=\sqrt{4 c_{2} \rho / M} k$, which can also be obtained in the GPE approach. The spin wave velocity is $v_{s}=\sqrt{4 \rho c_{2} / M}$.

However, only the vicinity of point $\mathbf{n}_{0}$ on the unit sphere is practically accessible in the representation employed in Ref. [32]. Eq.8, on the other hand, is valid for any point $\boldsymbol{\Omega}_{1} \sim \boldsymbol{\Omega}_{2}$ on the sphere. Therefore, the effective NL $\sigma \mathrm{M}$ derived below allows us to describe a spin correlated state far away from the one given in the GPE approach, as promised and provides at least a qualitative picture about the N-body ground state wave function well beyond the simple mean field solution. 


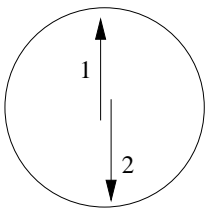

(a)

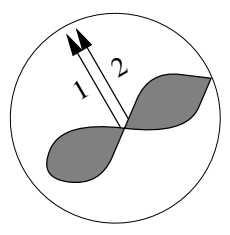

(d)

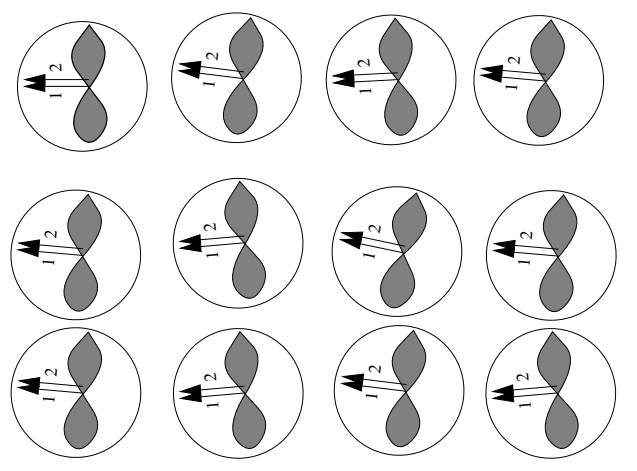

(f)

Fig.1 1) States represented by two unit vectors $\boldsymbol{\Omega}_{1}$ and $\boldsymbol{\Omega}_{2}$ ): a) $u^{2}$; b) $u v$; c) $v^{2}$. 2) $\boldsymbol{\Omega}_{1}$ and $\boldsymbol{\Omega}_{2}$ ) in zero field d), and in an external field $H$ e); 3) An example of microscopic wave functions of spin nematic states as indicated in $\mathrm{f}$ ). In a)-f), arrows stand for $\boldsymbol{\Omega}_{1,2}$; shapes of shaded blobs are given by $r=3|u(\boldsymbol{\Omega}) v(\boldsymbol{\Omega})|^{2}$ in a polar coordinate $(r, \theta, \phi)$. Note in d) the orientation of $\mathbf{n}=\left(\boldsymbol{\Omega}_{1}+\boldsymbol{\Omega}_{2}\right) / 2$ is arbitrary while in e) $\mathbf{n}$ is perpendicular to the external magnetic field.

The low frequency sector Lagrangian for the spin and the superfluid component can be derived by taking into account Eqs. 8.11

$$
\begin{aligned}
& \mathcal{L}=\mathcal{L}_{s}+\mathcal{L}_{c} \\
& \mathcal{L}_{c} \approx \frac{\rho}{2 M}\left[(\nabla \chi(\mathbf{r}))^{2}+\frac{1}{v_{c}^{2}}\left(\partial_{\tau} \chi\right)^{2}\right] \\
& \mathcal{L}_{s}=\frac{\rho}{2 M}\left[(\nabla \mathbf{n}(\mathbf{r}))^{2}+\frac{1}{v_{s}^{2}}\left(\partial_{\tau} \mathbf{n}\right)^{2}\right] .
\end{aligned}
$$

We introduce $\tau=i t$ as the imaginary time. The nonlinearlity is imposed via the constraint $\left|\mathbf{n}^{2}\right|=1$ at a low frequency limit. Eq.13 is the main result of the mapping and we keep terms which are of the lowest order in terms of $\partial_{\tau}$ and $\nabla . \mathcal{L}_{c}$ is taken in a Gaussian approximation and should be replaced by a full Gross-Pitaveskii Lagrangian in general. We will be mostly interested in the physics in the spin sector and simplification in $\mathcal{L}_{c}$ doesn't affect conclusions in this paper. $\mathcal{L}_{s}$ in Eq.13 represents an $o(3) \mathrm{NL} \sigma \mathrm{M}$; a coupling between the spin and superfluid component due to the Berry's phase effect has been neglected [37,38.

The partition function of the Lagrangian can be considered as the ground state wave function, with all the zero point fluctuations and their interactions included. The Lagrangian $\mathcal{L}_{s}$ has been been studied extensively before. Many fascinating properties of the spinor BEC therefore can be obtained by the mapping presented in this section. Depending on the ratio between two-body scattering lengths and interatomic distances, Eq.13 admits solutions with very different spin correlations. The symmetry broken states will be discussed in the next section and symmetry unbroken states are to be addressed in section IV.

\section{B. $Z_{2}$ symmetries and $Z_{2}$ gauge fields}

In the previous subsection, we have shown that the low energy spin dynamics of the BECs of spin one atoms with antiferromagnetic interactions is equivalent to that of the NL $\sigma \mathrm{M}$. These BECs support two branches spin wave excitations. In this subsection, we are going to illustrate an extra discrete symmetry enforced by the antiferromagnetic interactions. This discrete symmetry effectively couples the spin and phase degrees of freedom.

Let us first look at the the ground state wave function shown in Eq.7 with $\boldsymbol{\Omega}_{1,2}=\mathbf{n}$. It is invariant under a global transformation $\mathbf{n}, \chi \rightarrow-\mathbf{n}, \chi+\pi$; i.e.,

$$
\Psi(\mathbf{n}, \chi)=\Psi(-\mathbf{n}, \chi+\pi), \Psi(\mathbf{n})=(-1)^{N} \Psi(-\mathbf{n}),
$$

where $\chi$ is the phase of the scalar field $\Phi(x)$ introduced in Eq.7. In obtaining this symmetry, we notice $u(\mathbf{n})=\exp (i \pi / 2) v(-\mathbf{n})$, with $\pi / 2$ from a phase of a spin$\frac{1}{2}$ particle under a $180^{\circ}$ rotation.

This is certainly a quite general feature of all low lying excitations in ${ }^{23} \mathrm{Na}$ with antiferromagnetic interactions. It can also be illustrated using the zero mode Hamiltonian

$$
\mathcal{H}_{z . m .}=\rho c_{2} \frac{\mathbf{L}^{2}}{2 N},
$$

$\mathbf{L}$ is the total spin of the BEC. And

$$
\begin{aligned}
& {\left[\mathbf{n}_{\alpha}, \mathbf{n}_{\beta}\right]=0,} \\
& {\left[\mathbf{L}_{\alpha}, \mathbf{n}_{\beta}\right]=i \hbar \epsilon^{\alpha \beta \gamma} \mathbf{n}_{\gamma},\left[\mathbf{L}_{\alpha}, \mathbf{L}_{\beta}\right]=i \hbar \epsilon^{\alpha \beta \gamma} \mathbf{L}_{\gamma} .}
\end{aligned}
$$

Eqs.15, 16 show that $L$ is an angular moment operator defined on the unit sphere spanned by $\mathbf{n}$. In a spherical coordinate where $\mathbf{n}=(\sin \theta \cos \phi, \sin \theta \sin \phi, \cos \theta), \mathbf{L}$ is a differential operator with respect to $\theta, \phi$ 37. The eigenstates of the zero dimension Hamiltonian are spherical harmonics of the collective variable $\mathbf{n}$ 


$$
\Psi(\mathbf{n})=Y_{l, m}(\theta, \phi) .
$$

The energy spectrum is given by $\mathbf{L}^{2}=l(l+1)$, which is identical to that in [34]. The symmetry of Bosonic wave functions imposes further constraints on parities of the states: $l=0,2,4, \ldots . N$, if $N$ is an even number; and $l=1,3,5, \ldots$ otherwise. Eq. 17 shows the ground state is $l=0$ or a spin singlet when $N$ is an even number. For an odd $N$, the ground state has a threefold degeneracy with $l=1$. The collective behavior of the ground state depends on the even-oddness of the number of atoms in the BEC.

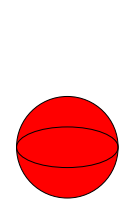

(a)

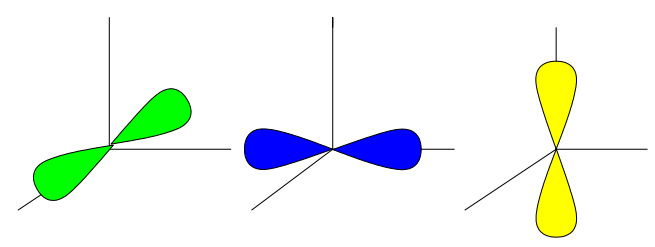

(b)
Fig.2 The ground states for an even a) and odd b) number of atoms in terms of the collective variable $\mathbf{n}$. In a), the ground state is a non-degenerate $s$-orbit while in b), the ground state has a threefold degeneracy corresponding to one of $p_{x}, p_{y}, p_{z}$ orbits, following Eq.17.

Now we are ready to construct low lying wave packets, taking into account the symmetry of Bosonic wave functions,

$$
\Psi_{N}(\mathbf{n})= \begin{cases}\sum_{l=0,2 \ldots} A_{l, m} Y_{l, m}(\mathbf{n}), \quad N \text { is even } \\ \sum_{l=1,3 \ldots} A_{l, m} Y_{l, m}(\mathbf{n}), \quad N \text { is odd }\end{cases}
$$

One easily verifies that the wave function written in Eq.18 observes the same $Z_{2}$ symmetry as a symmetry broken state. This is not surprising from the point of view that a symmetry broken state can be considered as a wave packet constructed out of the exact spectrum.

The effect of the $Z_{2}$ symmetry is manifestly dramatic on the dynamics of the spinor BEC. A full description of the $\mathrm{BEC}$ based on the $Z_{2}$ gauge fields was given in previous works, taking into account the entanglement of the order parameter space. So far, we haven't been able to impose this constraint of Eq.18 in the function integral in a continuous limit. However, by introducing an optical lattice, we are able to enforce the $Z_{2}$ symmetry in a mapping. A detail derivation was presented in a preprint by Demler et al. [42]; a short account of results was given by Demler and Zhou 43. I will illustrate the spirit of the derivation and quote the results here.

Let me describe an optical lattice where the dynamical effects of the discrete symmetry can be conveniently discussed [50,51,52,53, 54,55,56,57,18]. Experimentally, following Grynberg et al. [50], a body-center cubic structure can be created by having the following four laser beams interfere with each other: a circularly polarized light propagating along $z$ direction and three linearly polarized beams, with polarization plane coinciding with the $x y$ plane propagating in directions perpendicular to the $z$ axis. The lattice constant in an optical lattice is determined by the wavelength of the laser. In Gryberg et al.'s experiment, this is $514 \mathrm{~nm}$; the elasticity of the lattice is tunned by the laser intensity. So the model I am going to study is a realistic one from the experimental point of view. On the other hand, all the phenomena we are going to discuss should occur in both single traps and optical lattices, though it is perhaps more practical to observe them in a lattice setting. The feasibility was discussed in 42.

So we will have zero dimensional BECs with certain number of atoms living at each site described by Eq.15. subject to the constraint in Eq.18. The atoms can also hop between sites in the presence of quantum tunneling. The Hamiltonian of an array of identical optical traps is given by

$$
\begin{array}{r}
\mathcal{H}=\sum_{i} \mathcal{H}_{i}+\sum_{i j} \mathcal{H}_{i j} \\
\mathcal{H}_{i}=\frac{u}{2}\left(N_{i}-N_{0}\right)^{2}+\frac{g}{2} \mathbf{L}_{i}^{2}, \\
\mathcal{H}_{i j}=-2 J \mathbf{n}_{i} \mathbf{n}_{j} \cos \left(\chi_{i}-\chi_{j}\right)
\end{array}
$$

where

$$
\mathbf{L}_{i}=i \mathbf{n}_{i} \times \frac{\partial}{\partial \mathbf{n}_{i}}, N_{i}=\frac{\partial}{\partial \chi_{i}},
$$

and $J \approx t N$. The Hamiltonian given here also includes the phase degree of freedom to preserve the discrete symmetry under a discrete gauge transformation.

As indicated before, for each site characterized by the Hamiltonian in Eq.19, there are two sorts of elementary excitations: a)the excitations carrying no $L$ but with $N$; b)the excitations carrying no $N$ but with $L$. All excitations are classified by two quantum numbers $N$ and $L$ as $(n, l)$. The symmetry of the wave function observed by the low lying excitations is imposed by a constraint that the sum of $n$ and $l$ is an even integer. So only $(n, 2 l-n)$ are the physical excitations. For instance, a $(0,2)$ excitation corresponds to flip the spin of one of atoms without changing the number of the atoms; a $(2,0)$ excitation is to add a singlet pair to the condensate. And at last, a $(1,1)$ excitation represents adding one atom of spin one to the BEC.

To carry out a functional integral in the representation of $\left(n_{i}, l_{i}\right)$ while preserving the symmetry of the wave function, we use a projection operator

$$
\mathcal{P}_{i}=\frac{1}{2} \sum_{\sigma_{i}= \pm 1} \exp \left[i \frac{\pi}{2}\left(1-\sigma_{i}\right)\left(n_{i}+l_{i}\right)\right]
$$

that introduces a new Ising variable $\sigma_{i}= \pm 1$. The physical Hamiltonian therefore is 


$$
\mathcal{H}_{p h y}=\mathcal{H} \prod_{i} \mathcal{P}_{i} .
$$

Projecting an enlarged space $(n, l)$ into $(n, 2 m-n)$ therefore results in a set of discrete variables which were identified as the $Z_{2}$ gauge fields. The long wave length physics is characterized by the following action,

$$
\begin{array}{r}
S=-\sum_{r r^{\prime}} J_{r r^{\prime}}^{c} \sigma_{r r^{\prime}} \cos \chi_{r r^{\prime}}-\sum_{r r^{\prime}} J_{r r^{\prime}}^{2 c} \cos \left(2 \chi_{r r^{\prime}}\right) \\
-\sum_{r r^{\prime}} J_{r r^{\prime}}^{s} \sigma_{r r^{\prime}} \mathbf{n}_{r} \mathbf{n}_{r^{\prime}}-\sum_{r r^{\prime}} J_{r r^{\prime}}^{2 s} Q_{r}^{a b} Q_{r^{\prime}}^{a b}
\end{array}
$$

Here

$$
Q_{r}^{a b}=\mathbf{n}_{r}^{a} \mathbf{n}_{r}^{b}-\frac{1}{n} \delta^{a b}
$$

is a nematic order parameter for the $n$-component unit vector $\mathbf{n}(n=2$ when $\mathbf{n}$ lies in the plane and $n=3$ when it can rotate in all three directions). The coupling constants are $J_{r, r \pm \hat{\tau}}^{c}=(\epsilon u)^{-1}, J_{r, r \pm \hat{\tau}}^{s}=(\epsilon g)^{-1}$, $J_{r, r \pm\{\hat{x}, \hat{y}, \ldots\}}^{c, s}=\epsilon J|\eta|$, and $J_{r, r \pm \hat{\tau}}^{2 c}=J_{r, r \pm \hat{\tau}}^{2 s}=0$, $J_{r, r \pm\{\hat{x}, \hat{y}, \ldots\}}^{2 c, 2 s}=-\epsilon J / 4 . \epsilon$ is the time unit introduced when we discretize the time; $r=(i, \tau)$ and $r+\hat{\tau}=(i, \tau+\epsilon)$. And $\chi_{r r^{\prime}}=\chi_{r}-\chi_{r^{\prime}}$. Finally, $\eta$ is a parameter in the Hubbard-Stratanovich transformation [42,43].

The site variables $\exp (i \chi)$ and $\mathbf{n}$ live in $S^{1}$ and $S^{2}$ respectively, representing the phase and spin degree of freedom at each site. $\sigma_{r r^{\prime}}= \pm 1$ are discrete linking variables living in a $Z_{2}$ space. In the $S^{2}$ sector, we recovered the $\mathrm{NL} \sigma \mathrm{M}$ description of spin dynamics obtained in the previous sector. However, under the influence of the $Z_{2}$ symmetry, the spin-phase degrees of freedom are entangled, with interactions mediated by the $Z_{2}$ gauge fields.

It is obvious that the form of the action in Eq.23 is the simplest one consistent with the charge $U(1)$, spin $S O(3)$, and gauge $Z_{2}$ symmetries of the model. Another term that is allowed by the $Z_{2}$ symmetry and that is generated by integrating out the high energy degrees of freedom is the analogue of the Maxwell terms for the lattice gauge models

$$
S_{\sigma}=-K \sum_{\square} \prod_{\square} \sigma_{r r^{\prime}}
$$

where the summation goes over plaquettes in $d+1$ dimensional lattices. One can confirm that Eqs.23,25 are invariant under the following $Z_{2}$ gauge transformation

$$
\chi \rightarrow \chi+\pi, \mathbf{n} \rightarrow-\mathbf{n}, \sigma_{r r^{\prime}} \rightarrow-\sigma_{r r^{\prime}} .
$$

Note that the terms proportional to $J^{2 c, 2 s}$ are invariant under the inversion defined in Eq.26. That is, $\exp \left(i 2 \phi_{r}\right), Q_{r}^{a b}$ transform into themselves under the $Z_{2}$ transformation and carry zero $Z_{2}$ charges.

In the next few sections we will apply the mapping we obtained so far to explore some aspects of the BEC of spin one atoms with antiferromagnetic interactions. We start with the symmetry broken states.

\section{QUANTUM SPIN NEMATIC BECS}

The symmetry broken states of the BEC support very fascinating topological excitations, because of the peculiar structure of the internal space. In the next subsections, I will explore these excitations in some detail, emphasising on the influence of the $S^{2}$ and $Z_{2}$ symmetry in the spinor BEC.

\section{A. Topological defects in BECs}

The Lagrangian admits a symmetry broken solution when the two-body scattering is weak. Topological defects are of particular interest because of the symmetry in Eq.14. They also play very important roles in the quantum symmetry restoring and are vital for the complete understanding of thermal and quantum phase transitions. I will restrict myself to the static defects which can be directly detected in an experiment.

In the previous section, two parameters, $\exp (i \chi)$ and $\mathbf{n}$ are introduced for the description of BECs. The ground state is degenerate under a gauge transformation of the $U(1)$ group or a rotation of $\mathbf{n}$ on a unit sphere $S^{2}$. Furthermore, following Eq 14, the wave function is indistinguishable under a $180^{\circ} \mathbf{n}$ rotation and a gauge transformation: $\chi \rightarrow \chi+\pi$. Therefore, the internal space for the order parameter is $\mathcal{R}=\left[S^{1} \times S^{2}\right] / Z_{2} ; S^{1} \times S^{2}$ factorized with respect to $Z_{2}$ is the product space of a unit circle and a unit sphere with two diametrically opposite points identified. That is $(\chi, \mathbf{n})=(\chi+\pi,-\mathbf{n})$. This state should be defined as a quantum spin nematic state (QSNS).

The symmetry found in Eq.14 was not recognized in previous works and the internal order parameter space was misidentified as $U(1) \times S^{2}$ in [32], instead of [U(1)× $\left.S^{2}\right] / Z_{2}$ discussed here. On the other hand, for a classical nematic liquid crystal, the order parameter space is a unit sphere $S^{2}$ factorized with respect to $Z_{2}$. The new complication in the QSNS, which originates from the extra unit circle $S^{1}$ characterizing the superfluidity, plays a very important role in topological defects. 

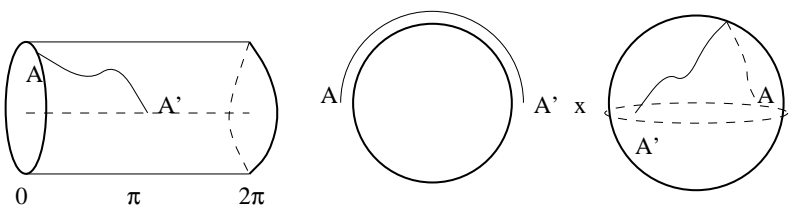

(a)
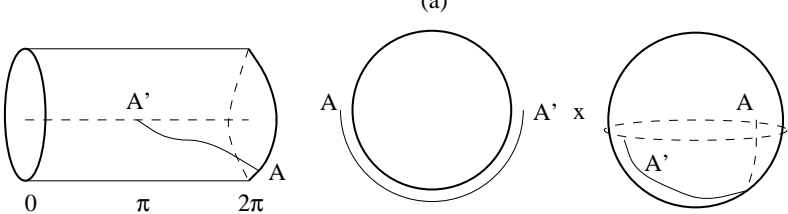

(b)
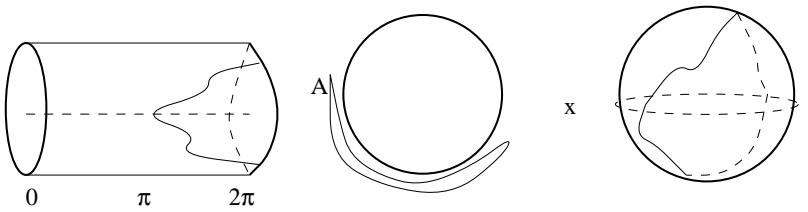

(c)
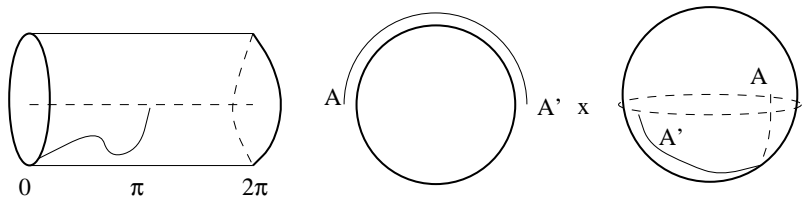

(d)
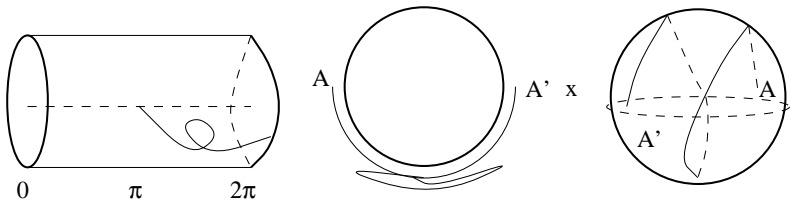

(e)

Fig.3. Examples of paths in $\mathcal{R}\left(\left[S^{1} \times S^{2}\right] / Z_{2}\right)$ and their projections in $S^{1}$ and $S^{2}$. $\left[S^{1} \times S^{2}\right] / Z_{2}$ is represented by a torus or a cylinder with ends identified. The axis of the cylinder stands for a unit circle, along which $\chi$ increases from 0 to $2 \pi$. Each disk, perpendicular to the axis, with boundary identified, is homotopic to a sphere; the boundary and the center of the disk are identified as the northern and southern pole of a twosphere. Points such as $A$ and $A^{\prime}$, which are diametrically opposite in the product space, are identical. The examples of paths homotopic to nonzero elements of $\pi_{1}\left(\left[S^{1} \times S^{2}\right] / Z_{2}\right)$ are shown in a) $(m=1, n=0)$ (see Eq.27), b) $(m=-1, n=-1)$. A path homotopic to zero element is shown in c) $(m=2, n=0)$. Paths in d) and e) are homotopic to paths in a) and b) respectively.

The linear or point defects in this case are determined by the classes of equivalent maps from a circle $S^{1}$ or a sphere $S^{2}$ of a real space into an internal space $\mathcal{R}$, following the general principle for the classification of defects in a symmetry broken state [58,59, 60]. The ensemble of these classes, or the fundamental and second homotopy group of space $\mathcal{R}$ are $\pi_{1,2}(\mathcal{R})$. Finally, textures, which correspond to defects with no singularities, are determined by a mapping from a three-sphere, a three dimensional space with surface identified, to $\mathcal{R}$. Correspondingly, the spin configuration is homogeneous at infinity. The homotopic group for textures is $\pi_{3}(\mathcal{R})$.

\section{1. $Z_{2}$ strings}

The fundamental homotopy group of space $\left[S^{1} \times\right.$ $\left.S^{2}\right] / Z_{2}$ is a product of an integer group $Z$ and a two element group $Z_{2}$. The elements of a $Z_{2}$ group are the indices for spin disclinations and $Z$ is the winding number of superfluid vortices. A closed path $\Gamma$ belonging to the nontrivial element of the $Z_{2}$ group is the one connecting two diametrically opposite points $(\chi, \mathbf{n})$ and $(\chi+\pi,-\mathbf{n})$ in the space $\left[S^{1} \times S^{2}\right]$. All other paths can either be deformed continuously into this one or into topologically trivial ones of $Z_{2}$ through escaping in a third dimension(see Fig. 3).

The corresponding wave function of linear singularities $\left(Z_{2}\right.$ strings) can be written as

$$
\begin{aligned}
& \Psi\left(\left\{\xi_{\alpha}\right\}\right)=\Pi_{\alpha} \Phi\left(\left\{\xi_{\alpha}\right\}\right) \\
& \left(\frac{\xi_{\alpha}-\xi_{0}}{\left|\xi_{\alpha}-\xi_{0}\right|}\right)^{n+w(m)}\left[\frac{1}{2} \operatorname{Re}\left(\frac{\xi_{\alpha}-\xi_{0}}{\left|\xi_{\alpha}-\xi_{0}\right|}\right)^{m / 2}\left(v^{2}-u^{2}\right)\right. \\
& \left.+\frac{i}{2} \operatorname{Im}\left(\frac{\xi_{\alpha}-\xi_{0}}{\left|\xi_{\alpha}-\xi_{0}\right|}\right)^{m / 2}\left(v^{2}+u^{2}\right)\right] \\
& \lim _{\xi \rightarrow \infty} \mathbf{n}(\xi)=\operatorname{Re}\left(\frac{\xi-\xi_{0}}{\left|\xi-\xi_{0}\right|}\right)^{m / 2} \mathbf{e}_{x}+\operatorname{Im}\left(\frac{\xi-\xi_{0}}{\left|\xi-\xi_{0}\right|}\right)^{m / 2} \mathbf{e}_{y} \\
& \lim _{\xi \rightarrow \infty} \mathbf{v}_{s}(\xi)=\frac{w(m)+n}{M\left|\xi-\xi_{0}\right|} \\
& {\left[\operatorname{Im}\left(\frac{\xi-\xi_{0}}{\left|\xi-\xi_{0}\right|}\right) \mathbf{e}_{x}-\operatorname{Re}\left(\frac{\xi-\xi_{0}}{\left|\xi-\xi_{0}\right|}\right) \mathbf{e}_{y}\right]}
\end{aligned}
$$

Here $\xi=x+i y ; m, n$ are integers and $w(m)=1 / 2$ for odd $m$ and $w(m)=0$ for even $m$. We assume lines located at $\xi=\xi_{0}=x_{0}+i y_{0}$. Each string is characterized in terms of $(m, n) . \mathbf{v}_{s}$ is defined as the superfluid velocity.

The $w(m)$ dependent part of $\mathbf{v}_{s}$ is present to ensure the single valuedness of the condensate wave function under the spin rotation. The spin wave function changes its sign when an atom moves along a $Z_{2}$ string given in Eq.27, following the identity $u(\mathbf{n}) v(\mathbf{n})=-u(-\mathbf{n}) v(-\mathbf{n})$. The final spin wave function differs from the initial one by a minus sign. In a string $(1,0), \mathbf{v}_{s}$ of a half vortex is present solely to compensate the $\pi$ phase under $\mathbf{n} \rightarrow-\mathbf{n}$ rotationally in a $Z_{2}$ string, as indicated in Fig.4. A nematic $Z_{2}$ string $(1, n)$ therefore corresponds to a $\pi$ spin disclination superimposed by a vortex with $n+1 / 2$ flux quantum, as shown in Fig.4 62].

This composite structure of linear defects is uniquely associated with the coherence of the condensate and is absent in a classical nematic liquid crystal. From an energetic point of view, a bare $\pi$-disclination carries a 
cut along which the phase changes abruptly from $\pi$ to $2 \pi$. This cut starting at the disclination ends only at the boundary of the coherent BEC and costs an energy linear in terms of the size of the system. For a similar reason, the energy cost to separate a half vortex and a $\pi$-disclination at distance $\mathrm{L}$ is linearly proportional to $L$. The composite strings shown in Fig.4 should be considered as a result of the confinement of $\pi$-disclinations and half vortices in the spinor BEC.

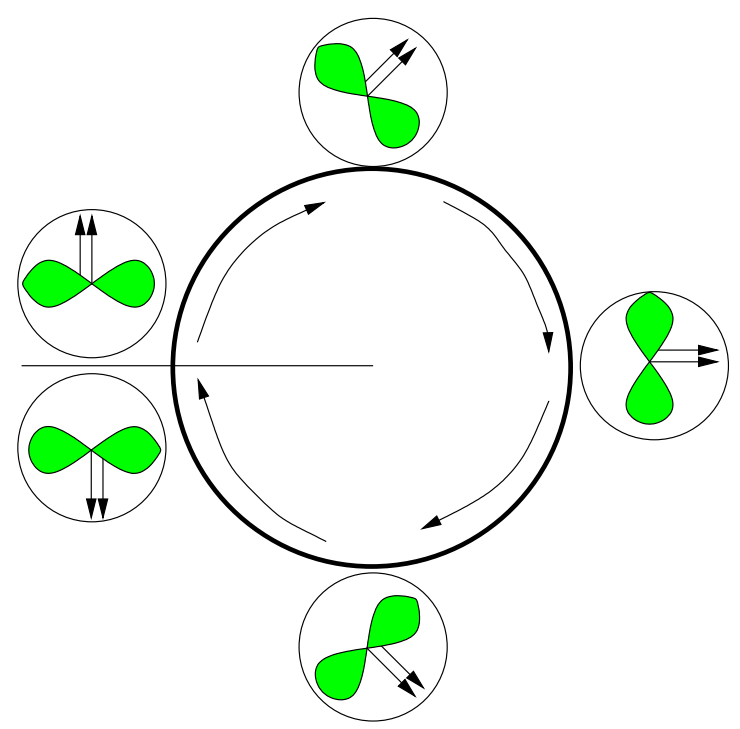

(a)
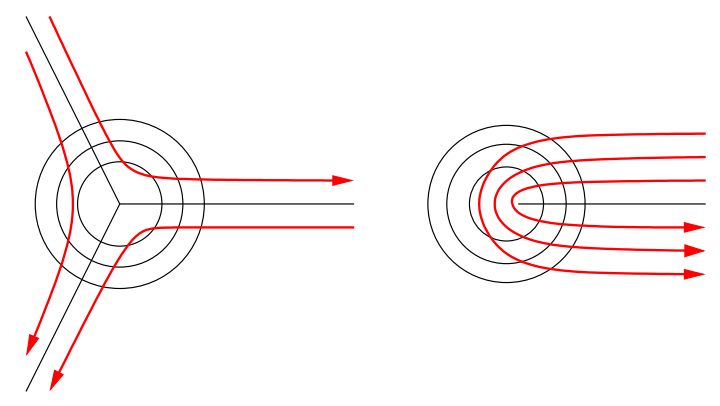

(b)

Fig.4 $Z_{2}$ strings: a) spin wave functions along a loop enclosing a defect (see also the caption of Fig.1); b) $\pi$ spin disclinations (defined in Eq.27) superimposed with superfluid vortices with half flux quanta (circular lines). In b), solid lines with arrows represent variation of $\mathbf{n}$ in defects.

The energy of a linear singularity $m=1, n=0$ is $\rho L / 4 M \ln (L / a)$, with $L$ as the system size. We should emphasis that the spin component of the wave function carries a zero supercurrent. And $\mathbf{v}_{s}$ is only determined by the polynomial of degree $n+w(m)$ in Eq.27.

$(-1, n),( \pm 3, n),( \pm 5, n), \ldots$ linear singularities can be obtained from $(-1, n)$ by continuous mappings(see ex- amples in Fig.3). For instance, $(-1, n)$ can be deformed into $(1, n)$ because of "an escape in a third dimension".

For an even number $m, \mathbf{n}$ follows a closed loop on a unit sphere as a spin moves along the linear defects. The Berry's phase caused by the spin rotation along the linear defect is zero in this case, following an identity

$$
\operatorname{Im} \int_{S} d \mathbf{n} \cdot \int \frac{d \Omega}{4 \pi} \frac{\partial}{\partial \mathbf{n}} u^{*}(\mathbf{n}) v^{*}(\mathbf{n}) \times \frac{\partial}{\partial \mathbf{n}} u(\mathbf{n}) v(\mathbf{n})=0 .
$$

The integral is carried over the area $S$ on the unit sphere of $\mathbf{n}$ bounded by a path along which $\mathbf{n}$ varies as one moves along the defect.

So there is no superflow of a half integer circulation superimposed when $m$ is an even number. And $\mathbf{v}_{s}$ vanishes in defects $(m, 0)$ with an even integer $m$. More important, $m= \pm 2, \pm 4, \ldots$ can be deformed into $m=0$ configuration as shown in Fig.3 and are homotopically identical. There is only one family homotopically distinct nematic spin disclination $(1, n)$.

It is particularly interesting to have a closed loop string. The far field wave function of a closed loop linear defect can be expressed in a compact form as

$$
\begin{aligned}
& \Psi\left(\left\{\xi_{\alpha}\right\}\right)=\Pi_{\alpha} \Phi\left(\left\{\xi_{\alpha}\right\}\right) u\left(\mathbf{n}\left(\left\{\xi_{\alpha}-\xi_{0}\right\}\right)\right) v\left(\mathbf{n}\left(\left\{\xi_{\alpha}-\xi_{0}\right\}\right)\right), \\
& \lim _{|\xi| \rightarrow \infty} \mathbf{n}(\xi, \theta)=\operatorname{Re}\left(\frac{\xi}{|\xi|}\right)^{ \pm 1 / 2} \mathbf{e}_{\rho}+\operatorname{Im}\left(\frac{\xi}{|\xi|}\right)^{ \pm 1 / 2} \mathbf{e}_{z} . \\
& \lim _{|\xi| \rightarrow \infty} \mathbf{v}_{s}(\xi, \theta)=0
\end{aligned}
$$

Here $\mathbf{e}_{\rho}, \mathbf{e}_{z}$ are unit vectors in cylindrical coordinates $(z, \rho, \theta)$; and $\xi=\rho+i z$. The circle $\xi=\xi_{0}$ is the center of the linear defect. The spin rotation in a constant $\theta$ plane leads to a half vortex; for a closed loop in Eq. 29, the half vortex line forms a closed ring along $\mathbf{e}_{\theta}$ direction and has no effect in the far field.

The stability of a closed loop is a rather subtle issue and deserves some attentions. It depends on competitions between the energy associated with the tension of the loop and the spin fluctuations which unfavor a singular structure. Under certain conditions, one indeed can show that a close loop structure is stablized [63].

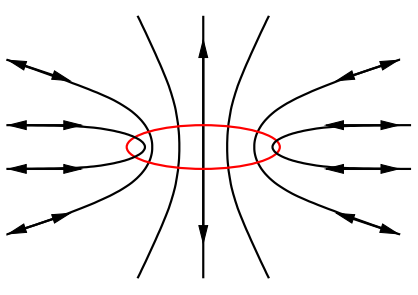

(a)

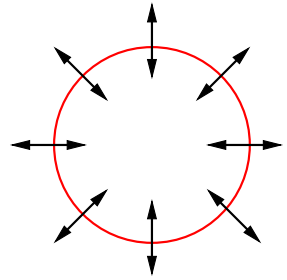

(b)
Fig.5 The far fields of a closed loop $\pi$-disclination are identical to those of a hedgehog. The topological charge of Pontryagin type spreads over the entire loop while in a hedgehog, the charge is localized at the center. 
However, if the loop shrinks, one ends up with a monopole which I am turning to in the next subsection. The intriguing connection between a closed loop $\pi$ disclination and a hedgehog also lies behind a recent interesting work [64]. In the non-Abelian field theory analogy of the situation discussed here, it was pointed out that a monopole could exist in a form of an Alice string. One can examine the connection field (defined in section VI) of a closed loop to show indeed the Pontryagin field strength is nonsingular at the center while the overall field strength at a large distance is still of a unit charge. The small distance behavior is distinct from a point-like defect. It implies that the unit charge of the Pontryagin type is uniformly distributed along the $\pi$ disclination. In a large loop limit, the linear charge density is vanishly small but with an integrated charge of unity. A charge exists as a closed loop string, with no singularities. Thus, the BECs of ${ }^{23} \mathrm{Na}$ might also be playgrounds for the physics of Alice strings. A close analogy of the Alice string physics was found in 45. where a hypermonopole is transformed into an antihypermonopole when moved along a $\pi$-disclination.

\section{Hedgehogs}

The second homotopy group $\pi_{2}\left(\left[S^{1} \times S^{2}\right] / Z_{2}\right)$ has nontrivial elements. Point defects are of the form of "hedgehogs" with the degree of mapping given by $N_{h}$,

$$
N_{h}=\frac{1}{4 \pi} \int d \theta d \phi \mathbf{n} \cdot \frac{\partial \mathbf{n}}{\partial \theta} \times \frac{\partial \mathbf{n}}{\partial \phi} .
$$

In a cylindrical coordinate $(z, \rho, \theta)$, the far field wave function of a $N_{h}=1$ hedgehog located at the origin is

$$
\begin{aligned}
& \Psi\left(\left\{\xi_{\alpha}\right\}\right)=\Pi_{\alpha} \Phi\left(\left\{\xi_{\alpha}\right\}\right)\left[\operatorname{Im}\left(\frac{\xi_{\alpha}}{\left|\xi_{\alpha}\right|}\right) u v+\right. \\
& \left.\frac{1}{2} \operatorname{Re} \frac{\xi_{\alpha}}{\left|\xi_{\alpha}\right|} \cos \left(\theta_{\alpha}\right)\left(v^{2}-u^{2}\right)+\frac{i}{2} \operatorname{Re} \frac{\xi_{\alpha}}{\left|\xi_{\alpha}\right|} \sin \left(\theta_{\alpha}\right)\left(v^{2}+u^{2}\right)\right] \\
& \lim _{\xi \rightarrow \infty} \mathbf{n}(\xi, \theta)=\operatorname{Re} \frac{\xi}{|\xi|} \mathbf{e}_{\rho}+\operatorname{Im} \frac{\xi}{|\xi|} \mathbf{e}_{z} \\
& \lim _{\xi \rightarrow \infty} \mathbf{v}_{s}(\xi, \theta)=0 .
\end{aligned}
$$

where $\xi=\rho+i z$.

However, consider a hedgehog with an index $N_{h}$. One can always move $\mathbf{n}(\mathbf{r})$ along a closed path $\Gamma$ in space $\left[S^{1} \times S^{2}\right] / Z_{2}$, which is homotopic to the nonzero element of $\pi_{1}\left(\left[S^{1} \times S^{2}\right] / Z_{2}\right)$. This connects $(\chi(\mathbf{r}), \mathbf{n}(\mathbf{r}))$ into $(\chi(\mathbf{r})+\pi,-\mathbf{n}(\mathbf{r}))$ and transforms a hedehog with index $N_{h}$ into a $-N_{h}$ hedgehog. In general, under the influence of $\pi_{1}\left(\left[S^{1} \times S^{2}\right] / Z_{2}\right)$, the elements in $\pi_{2}\left(\left[S^{1} \times S^{2}\right] / Z_{2}\right)$ can be transformed into one another (from $N_{h}$ into $-N_{h}$ ) by moving $(\chi, \mathbf{n})$ along the path $\Gamma$. This was emphasised in the investigation of a classical nematic liquid crystal in [59], where $\mathcal{R}=S^{2} / Z_{2}$. Physically it can be done by introducing a $\pi$ spin disclination superimposed by a $\pi$ vortex and moving the hedgehog along the linear defect; thus a linear singularity given in Eq.27 defines a continuous deformation from $N_{h}$ into $-N_{h}$. The point defect is characterized only by the modulus of $N_{h}$ and a hedgehog with an index $N_{h}$ is homotopically identical to that with $-N_{h}$. Following this discussion, the energy barrier involved in this deformation is the energy of the linear defect and is linear in terms of the sample size.

Despite the homotopical indistinguishability in the presence of linear defects, the positive and negative hedgehogs can be physically distinguished because of the coherence of the BEC, unlike the situation in a classical nematic liquid crystal. If a $\pi$ disclination is introduced alone, by moving a hedgehog around the linear defect, the condensate will acquire a $\pi$-phase with respect to the original one. The $\pi$-phase difference, which can manifest itself in a Josephson type of effect, is one of the signatures left behind by the positive or negative hedgehogs.

The second way to distinguish the positive and negative hedgehogs is to look at the local connection field. The idea here is to introduce a spin- $\frac{1}{2}$ collective excitation, which carries a half charge with respect to the connection fields. Unlike atoms which carry zero charges, in a positive hedgehog configuration, the spin- $\frac{1}{2}$ object experiences a connection field of an opposite sign compared with that of a negative hedgehog. This results in the Berry's phases of different signs in the positive and negative hedgehogs.

The other influence of the $Z_{2}$ symmetry on the point defect is that a hedgehog is identical to a closed loop $Z_{2}$ string as illustrated before. Though the spin hedgehog and the closed loop $\pi$ disclination are topologically identical, when spin fluctuations are insignificant the closed loop string has a higher energy because of the linear tension of a string. In this limit, we expect a closed loop, once created, collapses and leaves a spin hedgehog behind. The energy of a spin hedgehog is proportional to $\rho L / 4 M$, linear in terms of the system size $L$. Some static and dynamical aspects of the hedgehogs have also been investigated recently in 40].

The homotopical identity between a $\pi$-disclination and a hedgehog, and the conversion between the positive and negative hedgehog in the presence of $\pi$-disclinations are fascinating properties of the quantum spin nematic BEC. The $Z_{2}$ symmetry, after all, influences every aspect of the point defects under the consideration.

At this moment, we want to point out that the "hedgehog" in a ferromagnetic BEC is a "spin hedgehog" superimposed with a superflow. Along $-z$ axis, the circulation along $\mathbf{e}_{\theta}$ is quantized at $\int d \mathbf{l} \cdot \nabla \chi=1$, representing a vortex line ending at the monopole or a vorton discussed in [65, 59]. These features are absent in the QSNS for the reason that the Berry's phase as shown in Eq.28 is zero. 
Textures are characterized by a Hopf mapping from $S^{3}$ to $\left[S^{1} \times S^{2}\right] / Z_{2}$ in the presence of the superfluid component. In this case $\pi_{3}\left(\left[S^{1} \times S^{2}\right] / Z_{2}\right)=Z$ is an integer group. The Hopf number which characterizes the degree of mapping can be written in terms of $\mathbf{n}$ as

$$
\begin{aligned}
& H=\frac{1}{8 \pi} \int d^{3} \mathbf{r} \mathbf{A} \cdot \nabla \times \mathbf{A}, \\
& \nabla \times \mathbf{A}=\epsilon^{a b c} \epsilon_{i j k} \mathbf{n}_{a} \partial_{j} \mathbf{n}_{b} \partial_{k} \mathbf{n}_{c} \mathbf{e}_{i} .
\end{aligned}
$$

In a cylindrical coordinate $(\rho, z, \theta)$, the $\rho-z$ plane is homotopical to a two-sphere, with the northern pole identified as the center of the texture and the southern pole as the boundary. The configuration on a $\rho-z$ plane is therefore homotopically identical to a mapping from $S^{2}$ to a $\left[S^{1} \times S^{2}\right] / Z_{2}$ and can be considered as a Skyrmion on the $\rho-z$ plane. By rotating it along $z$ axis, we obtain the texture with Hopf number $H=1$. The center of the texture is located along a circle $\xi=\xi_{0}$ around $z$ axis $(\xi=\rho+i z)$. Similarly, surface $\operatorname{Im}\left(\xi-\xi_{0}\right) / \operatorname{Re}\left(\xi-\xi_{0}\right)=$ const with boundaries identified is also homotopically identical to a two sphere $S^{2}$. Under the Hopf mapping, the spin configurations on these planes are the same as Skyrmions.

So in a cylindrical coordinate $(z, \rho, \theta)$, the far field wave function for a texture with $H=1$ is

$$
\begin{aligned}
& \Psi\left(\left\{\xi_{\alpha}\right\}\right)=\Pi_{\alpha} \Phi\left(\left\{\xi_{\alpha}\right\}\right) \\
& {\left[\frac{1}{2} \sin g\left(\left|\xi_{\alpha}-\xi_{0}\right|\right) \operatorname{Re}\left(\frac{\xi_{\alpha}-\xi_{0}}{\left|\xi_{\alpha}-\xi_{0}\right|} \exp \left(i \theta_{\alpha}\right)\right)\left(v^{2}-u^{2}\right)\right.} \\
& +\frac{i}{2} \sin g\left(\left|\xi_{\alpha}-\xi_{0}\right|\right) \operatorname{Im}\left(\frac{\xi_{\alpha}-\xi_{0}}{\left|\xi_{\alpha}-\xi_{0}\right|} \exp \left(i \theta_{\alpha}\right)\right)\left(v^{2}+u^{2}\right) \\
& \left.+\cos g\left(\left|\xi_{\alpha}-\xi_{0}\right|\right) u v\right] \\
& \lim _{\xi \rightarrow \infty} \mathbf{n}(\xi, \theta)=\sin g\left(\left|\xi-\xi_{0}\right|\right)\left[\operatorname{Re} \frac{\xi-\xi_{0}}{\left|\xi-\xi_{0}\right|} \mathbf{e}_{\rho}+\operatorname{Im} \frac{\xi-\xi_{0}}{\left|\xi-\xi_{0}\right|} \mathbf{e}_{\theta}\right] \\
& +\cos g\left(\left|\xi-\xi_{0}\right|\right) \mathbf{e}_{z}, \\
& \lim _{\xi \rightarrow \infty} \mathbf{v}_{s}(\xi, \theta)=0
\end{aligned}
$$

Here $g(|\xi|=0)=0$ and $g(|\xi|=\infty)=\pi$. Once again, the superflow $\mathbf{v}_{s}$ is zero in a Hopf texture in the QSNS by contrast to that in a ferromagnetic BEC.

Since the order parameter is homogeneous at the boundary of the space, the texture can considered as a "particle" of a finite extend $\xi_{0}$. The energy is estimated as $\rho \xi_{0} / 4 M$ and decreases when the texture shrinks; this implies an instability towards collapsing. It can be energetically stablized only when high derivative terms are taken into account, as suggested in [66]. However, this is beyond the validity of the nonlinear sigma model where only the long wave length dynamics is correctly characterized.
In the presence of an external magnetic field along $z$ direction, $\boldsymbol{\Omega}_{1,2}=\mathbf{n} \pm \mathbf{e}_{z} g \mu_{B} H / 4 c_{2} \rho$, following Eq.11;

$$
\mathbf{l}=\frac{g \mu_{B} H}{4 c_{2} \rho} \mathbf{e}_{z} .
$$

The Lagrangian is the same as that without an external field (up to a constant term). However, the constraint that $\mathbf{n}$ has to satisfy depends on the external field. In the absence of an external magnetic field, Eq.9 is automatically satisfied for $\mathbf{l}=\mathbf{n} \times \partial_{t} \mathbf{n} / 4 c_{2}$. $\mathcal{L}_{s}$ is of the form of the $O(3)$ NL $\sigma \mathrm{M}$. An external field breaks the $S^{2}$ symmetry and confines the low frequency sector of $\mathbf{n}$ in a plane perpendicular to $\mathbf{H}$ itself, i.e.

$$
\mathbf{n} \cdot \frac{\mu_{B} H}{4 c_{2} \rho} \mathbf{e}_{z}=0 .
$$

The Lagrangian in the presence of a magnetic field is that of an $O(2) \mathrm{NL} \sigma \mathrm{M}$; it has the $S^{1}$-symmetry at the frequency $\omega \ll \mu_{B} H$. At the high frequency limit, following the equation of motion, $\mathbf{n}$ precesses in a field $4 c_{2} \mathbf{l}(x)$, much larger than the external one $H$ and the $S^{2}$-symmetry is restored.

As a consequence, if an external magnetic field $\mathbf{H}=$ $H \mathbf{e}_{z}$ is applied, $\mathbf{n}_{0}$ is in the $x y$ plane and the parameter space for $\mathbf{n}_{0}$ is a unit circle. The ground state is degenerate under the group $\left[U(1) \times S^{1}\right] / Z_{2}$, following Eq.13. The order parameter space for the quantum spin nematic state therefore is $\mathcal{R}=\left[S^{1} \times S^{1}\right] / Z_{2}$, which represents the product space of two unit circles but again with two diametrically opposite points $(\chi, \mathbf{n})$ and $(\chi+\pi,-\mathbf{n})$ being identical.

The fundmental group $\pi_{1}\left(\left[S^{1} \times S^{1}\right] / Z_{2}\right)$ has nontrivial elements. The corresponding wave function for linear defects can be written in the same form as that in Eq.27, $m, n$ are integers. However in this case, all defects $(m, n)$ are topological distinct (at energy scales lower than the external field). $m= \pm 1$ and $n= \pm$ are positive and negative $\pi$ spin disclinations, shown in Fig. 2 .

So far we discuss the static properties of the bulk defects. The dynamical aspects of these defects as well as surface defects will be addressed elsewhere. We also notice that in the presence of the external field, by contrast to the classical nematic liquid crystals, $\mathbf{n}$ in the QSNS is not parallel to the external field. This results in soundlike collective modes even in the presence of an external field, but with number of modes reduced from two(at zero field) to one.

We have restricted ourselves to the weak magnetic field limit and neglected the possible quadratic Zeemann shift $\mathcal{H}_{Q Z}=\sum_{\alpha} Q H^{2} F_{z \alpha}^{2}$ (the external field $H$ is along $\mathbf{e}_{z}$ direction). An inclusion of the quadratic Zeemann shift yields an additional term $\mathcal{L}_{Q Z}=\int \rho Q H^{2}\left(\mathbf{n}_{x}^{2}+\mathbf{n}_{y}^{2}\right) d x$ to the NL $\sigma \mathrm{M}$ derived in section II. The main effect of this 
contribution is to align $\mathbf{n}$ along the external field. Therefore, when this shift dominates, the ground state is left with a double degeneracy: $\mathbf{n}=\mathbf{e}_{z}$ and $\mathbf{n}=-\mathbf{e}_{z}$. The spin wave develops an energy gap of the order $Q H^{2}$. We will focus on the zero magnetic field case in the rest of discussions.

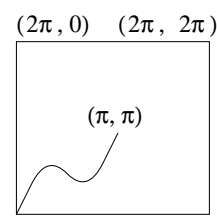

$(0,0) \quad(0,2 \pi)$
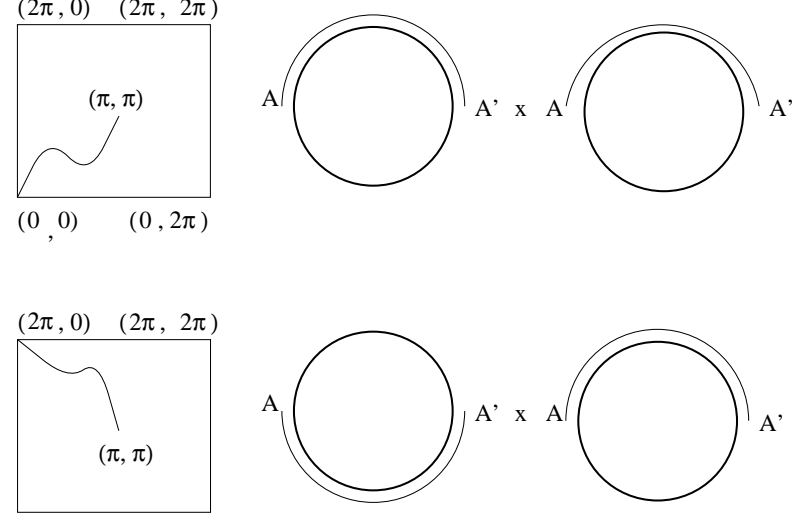

$\left(\begin{array}{ll}0 & 0) \\ (0,2 \pi)\end{array}\right.$
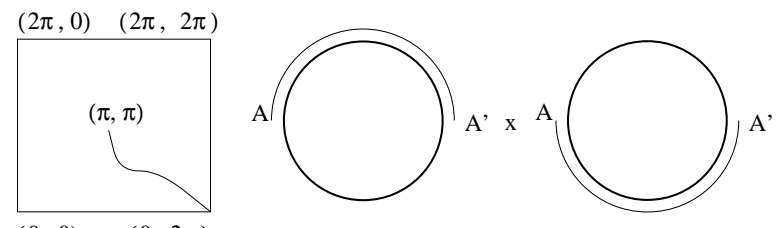

$(0,0) \quad(0,2 \pi)$
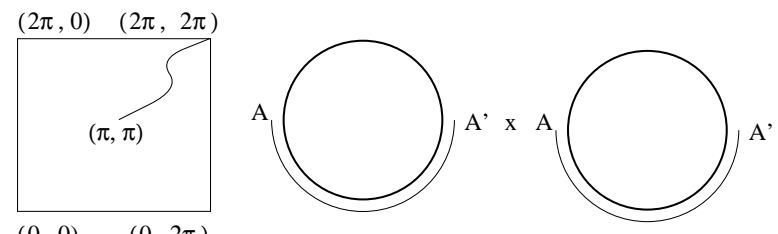

Fig.6. Examples of closed paths in $\mathcal{R}=\left[S^{1} \times S^{1}\right] / Z_{2}$ and projections in $S^{1}$ and $S^{1}$. Here the surface of a torus-2, or a square with boundaries parallel to each other identified, stands for $\left[S^{1} \times S^{1}\right] / Z_{2}$. The points such as $(\pi, \pi)$ and $(0,0)$, or $(0,2 \pi)$, or $(2 \pi, 2 \pi)$, or $(2 \pi, 0)$ are identical, representing two diametrically opposite points in the product space $S^{1} \times S^{1}$. The examples of paths which are homotopic to nonzero elements of $\pi_{1}\left(\left[S^{1} \times S^{1}\right] / Z_{2}\right)$ are shown in a). $(\mathrm{m}=1, \mathrm{n}=0), \mathrm{b}) .(\mathrm{m}=1, \mathrm{n}=-1), \mathrm{c}) .(\mathrm{m}=-1$, $\mathrm{n}=1), \mathrm{d}) .(\mathrm{m}=-1, \mathrm{n}=0)$. All are topologically distinct.

\section{SPIN DISORDERED QUANTUM CONDENSATES}

For the quantum nematic state considered in the previous sections, the full Hamiltonian includes $\mathcal{H}_{s}$ for the spin sector $\left(S^{2}\right), \mathcal{H}_{c}$ for the superfluid sector $\left(S^{1}\right)$, the coupling between two sectors $\mathcal{H}_{s c}$ and the constraint that the wave function remains invariant under a local $Z_{2}$ transformation, i.e., $\Psi(-\mathbf{n}(\mathbf{x}), \chi+\pi)=\Psi(\mathbf{n}(\mathbf{x}), \chi)$. To study quantum nematic fluctuations or quantum spin nematic order-disordered transitions, one has to integrate out the phase degree of freedom (superfluid component) under the constraint and obtain a renormalized spin dynamics.

While a full treatment of the quantum spin nematic fluctuations taking into account the influence of the $Z_{2}$ symmetry will be present in the next section, here I will be interested in a spin disordered condensate due to strong interactions between spin wave excitations in the $o(3) \mathrm{NL} \sigma \mathrm{M}$; in this limit, the coupling between the phase fluctuations and the spin fluctuations is irrelevant. The procedure is carried out self-consistently: first we obtain results based on the $\mathrm{o}(3) \mathrm{NL} \sigma \mathrm{M}$ and then consider the influence of the $S^{1}$ sector and the $Z_{2}$ symmetry. The reduction from $\left[S^{1} \times S^{2}\right] / Z_{2}$ to $S^{2}$ for the spin dynamics is possible based on the following considerations.

First, the phase fluctuations in the BEC and the nematic order fluctuations can affect each other via a direct coupling $\mathcal{L}_{s c}$. Nevertheless, the inclusion of $\mathcal{H}_{s c}$ only results in higher derivative terms; the coupling thus becomes important only at a rather high frequency $\hbar \rho^{2 / 3} / 2 M$, which is also the upper cut-off energy of the $\mathrm{NL} \sigma \mathrm{M}$.

Second, the constraint in Eq.14 leads to another coupling of a pure topological origin; for instance in the static limit, it leads to superimposed $Z_{2}$ strings as discussed in section III. The spin nematic order-disorder transition depends on the $Z_{2}$ gauge fields and spin waves in the $S^{2}$ sector. However, if the phase fluctuations are weak across the nematic order-disordered transitions driven by the zero point rotations of each atom, the $Z_{2}$ fields are effectively frozen and irrelevant to the discussion.

Indeed, in $1+1$ dimension discussed in the next section, the nematically disordered state exists in the weakly interacting limit where the phase fluctuations are still negligible. In terms of quantum tunneling, the nematic disordered state in $1+1$ dimension is driven by texture instantons. Quantum tunneling of the $Z_{2}$ strings (or $Z_{2}$ instantons) turns out to play little role in the nematic order-disordered transitions because of the coherent phase sector. This again implies the $Z_{2}$ gauge fields are not relevant to our discussion. In $3+1$ dimension, this is also the case and the transitions are not driven by the $Z_{2}$ gauge fields if the phases of the condensates remain coherent around the nematic order-disorder transition point.

As in other symmetry broken states, quantum fluctuations in $\mathbf{n}$ exist in the spinor BEC because $\mathbf{n}$ doesn't commute with the Hamiltonian and is not a conserved quantity. The quantum fluctuations in the QSNS can be studied by considering the NL $\sigma \mathrm{M}$,

$$
\mathcal{L}_{s}=\frac{1}{2 f}\left(\partial_{\mu} \mathbf{n}\right)^{2}, \mathbf{n} \cdot \mathbf{n}=1 .
$$


Here

$$
f=(16 \pi)^{1 / 2}\left(\rho \Delta a^{3}\right)^{1 / 6}, \Delta a=\frac{a_{2}-a_{0}}{3}
$$

in a single trap limit. We also introduce dimensionless length and time: $\tilde{\mathbf{r}}=\mathbf{r} \rho^{1 / 3}, \tilde{\tau}=\tau v_{s} \rho^{1 / 3}$, and $v_{s}=$ $\sqrt{4 c_{2} \rho / M}$. Derivatives $\partial_{\mu}$ are defined as $\left(\partial_{\tilde{\tau}}, \partial_{\tilde{x}}, \partial_{\tilde{y}}, \partial_{\tilde{z}}\right)$.

In the typical low frequency fluctuations or spin waves, $|\mathbf{L}|$ is much less than unity and each individual atom remains to be in a spin state $u(\mathbf{n}(\mathbf{r})) v(\mathbf{n}(\mathbf{r}))$, but with $\mathbf{n}(\mathbf{r}, t)$ varying in the space and time. The quantum fluctuations of the nematic order can be estimated in the lowest order approximation in the weakly interacting limit. This is similar to the spin wave approximation made for Heisenberg antiferromagnetic spin systems(HAFS) [67. The correlation function of $\delta Q^{\alpha \beta}=Q^{\alpha \beta}-<Q^{\alpha \beta}>$ $(\alpha=x, y, z)$ in the QSNS can be calculated as

$$
\begin{aligned}
& <\delta Q^{\alpha \beta}(\mathbf{r}, t) \delta Q^{\alpha^{\prime} \beta^{\prime}}(0,0)>= \\
& \sum_{\eta}\left(\delta_{\alpha 0} \delta_{\alpha^{\prime} 0} P_{\beta \eta} P_{\beta^{\prime} \eta}+\delta_{0 \beta} \delta_{0 \beta^{\prime}} P_{\alpha \eta} P_{\alpha^{\prime} \eta}\right. \\
& \left.+\delta_{0 \alpha} \delta_{0 \beta^{\prime}} P_{\beta \eta} P_{\alpha^{\prime} \eta}+\delta_{0 \beta} \delta_{0 \alpha^{\prime}} P_{\alpha \eta} P_{\beta^{\prime} \eta}\right) \mathcal{C}_{\eta}(\mathbf{r}, t) .
\end{aligned}
$$

$<>$ represents an average over all configurations. $\mathcal{C}_{\eta}=<$ $\delta \mathbf{n}_{\eta}(\mathbf{r}, t) \delta \mathbf{n}_{\eta}(0,0)>$ reflects the dynamics of the "director"; $\eta= \pm 1$ label eigen modes and 0 labels the direction of $\mathbf{n}_{0}=\mathbf{e}_{z} \cdot P_{\alpha \eta}=\mathbf{e}_{\alpha} \cdot \mathbf{e}_{\eta}$, where $\mathbf{e}_{\eta}$ is the unit vector of the $\eta$ th eigenmode; for $\mathbf{n}_{0}=\mathbf{e}_{z}, \mathbf{e}_{\eta}=\left(\mathbf{e}_{x}+i \eta \mathbf{e}_{y}\right) / \sqrt{2}$. We assume $\delta \mathbf{n}=\mathbf{n}-\mathbf{n}_{0}$ is small and different modes interact weakly. Following Eq.36,

$$
\mathcal{C}_{\eta}(\mathbf{r}, t)=f \int d \mathbf{P} d \omega \frac{\exp (i \mathbf{p} \tilde{x}-i \omega \tilde{t})}{\omega^{2}-\mathbf{p}^{2}}
$$

$\omega, \mathbf{p}$ are dimensionless variables. The amplitude of $\mathcal{C}_{\eta}$ is proportional to the parameter $f$. Eq. 39 is valid when $f$ is much less than unity. When $f$ increases, spin wave excitations start to interact strongly and Eq. 39 becomes invalid.

$f$ is a measure of the amplitude of quantum fluctuations in the BEC of ${ }^{23} \mathrm{Na}$. Following Eq 8, the energy of the system consists of two parts. a) The potential energy $\hbar^{2} \rho(\nabla \mathbf{n})^{2} / 2 M$, which is the energy cost in the presence of a slow variation of $\mathbf{n}$, aligns $\mathbf{n}$ of different atoms. It determines the bare spin stiffness. And b) the zero point kinetic(rotation) energy $\hbar^{2} \mathbf{l}^{2} / 2 I_{0}$, where $I_{0}=\left(4 c_{2} \rho / \hbar^{2}\right)^{-1}$ can be considered as the effective inertial of an individual atom. This rotation energy originates from the twobody scattering in the microscopic Hamiltonian and the inertial is inversely proportional to the scattering length. The zero point rotations tend to disrupt the order of $\mathbf{n}$ between different atoms. $f^{-1}$ is a square root of the ratio between the potential energy at an interatomic scale $\hbar^{2} \rho^{2 / 3} / 2 m$ and the zero point kinetic(rotation) energy $\hbar^{2} / 2 I_{0}$ of an individual atom.

\section{A. Rotation symmetry restored correlated states in 3d}

At zero temperature the $o(3) \mathrm{NL} \sigma \mathrm{M}$ in Eq. 36 has ordered and disordered quantum phases at $d>1$, depending on the parameter $f$. The renormalization group (RG) equation flow is determined by the interactions between collective modes. The scatterings between these spin waves are determined by the local nonlinear spin dynamics, especially spin waves around some slowly varying spin configurations. In the following, I will take this RG equation point of view of the NL $\sigma \mathrm{M}$ : $f$ is the quantity determining spin correlations in the ground state of the BECs.

The RG equation for $f$ can be obtained by first integrating out fluctuations within $e^{-l}<|\mathbf{p}|<1, e^{-l}<\epsilon<1$ and then rescaling $\mathbf{p} \rightarrow \mathbf{p} e^{l}, \epsilon \rightarrow \epsilon e^{l}$. In $3+1$ dimension, the $\mathrm{RG}$ equation takes a form

$$
\frac{d f}{d l}=-2 f\left(f_{c}-f\right)
$$

Within the framework of the NL $\sigma \mathrm{M}, f_{c}=8 \pi^{2}$ in $d=$ 3 (see Appendix B). However, the exact value of $f_{c}$ is determined by the details of the short-range behavior of the system and should be obtained only by numerics. The state where the long-range nematic order is absent due to zero point motions of collective variables is called a "spin disordered quantum condensate" (SDQC), to be distinguished from the "QSNS" where the long-range order is present.

At the strongly interacting or a high density limit $f>f_{c}$, the zero point kinetic energy dominates and the spin stiffness is renormalized to zero at a long wave length limit. Especially, in an extremely quantum disordered phase where the scatterings between two atoms are strong and the inertial is small, $\mathbf{n}$ of each atom rotates independently and the spin wave function of atoms only correlates at an interatomic distance. $\mathbf{n}$ of each spin experiences fast diffusive motion on the unit sphere $S^{2}$ with a mean free time $1 / 2 c_{2} \rho$ much shorter than $2 M / \hbar^{2} \rho^{2 / 3}$, due to the strong zero point rotation. The excitation spectrum has a gap of order of $2 c_{2} \rho$.

As the two-body scatterings get weaker, the inertial of each individual atom $I_{0}$ becomes higher and the motion of $\mathbf{n}$ on the unit sphere is slower because of a lower zero point rotation energy. Thus, $\mathbf{n}$ of different atoms starts to correlate at a finite length $\xi$, which is a function of the parameter $f$, much longer than the interatomic distance. As this happens, the resultant effective inertial increases proportional to the number of atoms within the range of the correlation length, $I(\xi) \sim \xi^{3} / 2 c_{2}$. And the spins of different atoms precess a collective temporal motion on the unit sphere with the mean free time $\tau$. Following Eq.8, $\mathbf{L}(\xi) \sim \xi^{3} / c_{2} \tau$ and the total rotation energy 
is $\mathbf{L}(\xi)^{2} / 2 I(\xi)$. This has to be balanced by the potential energy $\rho \xi / M$, leading to $\tau \sim \xi / \sqrt{c_{2} \rho / M}$. It implies that in this spin correlated state, collective excitations propagate with a bare spin wave velocity $\sqrt{c_{2} \rho / M}$ up to a scale $\xi$. Finite-range nematic order exists in this case and the correlation length can be estimated using the renormalization approach. In the current situation, $\xi=\rho^{-1 / 3} f_{c} /\left[\left(\rho \Delta a^{3}\right)^{1 / 6}-f_{c}\right]$. Local nematic order is established up to a distance $\xi$ in the BECs but the state is rotationally invariant. A phase transition takes place at $\rho \Delta a^{3}=8 \times 10^{-6} \times f_{c}^{6}$.

At weakly interacting or a low density limit $f<f_{c}$, the potential energy dominates and the spin stiffness flows to a finite value under the renormalization group transformation. Long-range order occurs. All spin one Bosons rotate as a rigid body with an effective inertial $I(N)=N / c_{2} \rho$. The energy gap in the excitation spectrum decreases accordingly

$$
E_{g a p} \propto \frac{\hbar^{2}}{2 I(N)}=\frac{\hbar^{2} c_{2} \rho}{2 N}
$$

as the number of Bosons $N$ increases and vanishes as a thermal dynamic limit is approached. The rotation symmetry is broken in the ground state of the BEC as nematic long-range order is established.

We want to make the following remarks concerning the statements made so far:

1). The quantum spin order-disorder transition in $3 d$ is driven by strongly interacting short wave length excitations. Therefore, in principal, it is no longer valid to neglect the coupling between spin and superfluidity as we did for the low frequency sector. We, however, believe the picture present here is a qualitative correct one and the coupling can at most shift the value of $f_{c}$.

$2)$. In the weakly disordered limit, the linear tension of a $Z_{2}$ string at a scale $L$ is of order $L \rho^{-1 / 3} E_{\text {core }}$, with the core energy $E_{\text {core }}$ of order $\hbar^{2} \rho^{2 / 3} / 2 m$. Large loop $Z_{2}$ instantons are extremely rare and can be neglected. This justifies the reduction we made at the beginning: The $Z_{2}$ gauge fields fluctuate weakly in this limit.

3) At a high density limit, one should also take into three-body, four-body scatterings. This can further modify the short distance dynamics but will not affect the conclusions arrived above in a qualitative way.

4) One should be cautious about the definition of "phase" since the alkali atoms under investigation are in a long lived metal stable gaseous state. The life time of atomic gas is limited by three-body inelastic collisions [7]. The collision rate increases dramatically as the density increases. For sodium atoms, the life time of the metal stable gas is given as [7] $\tau_{L}=10^{-12} \times\left(\rho \Delta a^{3}\right)^{-2} \times 100$ secs . If a reasonable experiment measurement can be performed at a time scale $10 \mathrm{~ms}$, then $\rho \Delta a^{3}$ should be less than $10^{-4}$. This sets a limit on $f_{c}$ in order for the quantum disordered nematic phase to be probed under the current experimental conditions. For $\rho \Delta a^{3}=10^{-6}$ as is in the experiments, long-range nematic order should be observed.

\section{B. Rotation symmetry retored states in $1 \mathrm{~d}$}

Quantum fluctuations of collective variables are most prominent in BECs confined in highly anisotropic traps. The $\mathrm{N}$ spin one bosons ground state wave function in this limit does not live in the zero momentum sector of the Hilbert space. The NL $\sigma \mathrm{M}$ turns out to be the most powerful approach to capture spin correlations in a rotationally invariant ground state. For simplicity, we approximate highly anisotropic traps as $1 d$ nematic BECs.

In $1 d$ traps at zero temperature and zero field, one should expect there will be no long-range order and the state is nematically disordered, following the renormalization group results of the NL $\sigma \mathrm{M}$ 68]. The RG equation in this case can be shown as

$$
\frac{d f}{d l}=\frac{1}{2 \pi} f^{2}
$$

which always flows into a strong coupling fixed point (disordered state) at the low energy and long wave length limit. The correlation length is $\xi=\rho^{-1} \exp (4 \pi / f)$ ( $\rho$ is the linear density). That is, in a 1d trap, the excitation spectrum should have an energy gap following [41].

In the $1 \mathrm{~d}$ spin nematic BEC under consideration, where the long wave length spin wave fluctuations are most significant and $\mathbf{l}(x) \sim 0, \mathcal{H}_{s c}$ can be effectively taken to be zero. To a good approximation, the phase (or density) fluctuations and the nematic spin fluctuations are indeed decoupled (the $Z_{2}$ gauge fields be also frozen as discussed at the end of this section). Therefore, the ground state can be spin disordered but phase coherent. The 1d BECs of ${ }^{23} \mathrm{Na}$ have long-range order in $\chi$ but short-range correlations in $\mathbf{n}$ in the weakly interacting limit.

These spin disordered states mimic the quantum spin liquid states proposed in the literature of Heisenberg antiferromagnetic systems (HAFS). They represent nematic liquids, where $\mathbf{n}$ of ${ }^{23} \mathrm{Na}$ atoms in the BEC aligns toward a certain direction up to a length scale $\xi$ and the order is disrupted afterwards due to the zero point motions originating from the spin-dependent two-body scatterings. The zero point motions in $\mathbf{n}$ also lead to quantum tunneling of spin textures, with the tunneling rate per $\xi$ inversely proportional to the energy gap.

We notice that the NL $\sigma \mathrm{M}$ obtained from the microscopic Hamiltonian in this paper doesn't have a $\theta$-term

$$
\mathcal{L}_{\theta}=\frac{\theta}{4 \pi} \int d \tau d x \mathbf{n} \cdot \frac{\partial \mathbf{n}}{\partial \tau} \times \frac{\partial \mathbf{n}}{\partial x},
$$

which is generally present in the HAFS studied before 41. The presence of a $\theta$-term in the NL $\sigma \mathrm{M}$ can dramatically affect the RG flow. A $\theta$-term with $\theta=\pi$, as in 
the half-integer HAFS, results in an additional term in the RG equation 69. In fact, the additional term arising from a $\theta$-term forces the RG to flow into a massless fixed point in $1 d$ and implies a power law decay of the correlation function or gapless excitations.

The absence of a $\theta$-term in the NL $\sigma \mathrm{M}$ derived here, which prevents the RG from flowing into a massless fixed point and ensures an energy gap in the excitation spectrum of the nematic state, can also be justified on a ground of the $Z_{2}$ symmetry. It follows a general fact that the Berry phase in an ordered state vanishes as a result of cancellation between $u(\mathbf{n})$ and $v(\mathbf{n})$ contributions, as shown in Eq.28.

We should note that in a nematic state, the textures with 1 and -1 indices in $1+1$ dimension are homotopically indistinguishable under the influence of the $Z_{2}$ symmetry. This is similar to what happens to the point defects in $3 d$ : the spin hedgehogs with index $N_{h}$ and $-N_{h}$ are indistinguishable as emphasised in section IV.

The $Z_{2}$ symmetry in our theory also leads to $\pi$ spin disclinations superimposed with half vortices in $1+1$ dimension: $Z_{2}$ instantons. Unlike texture instantons, the action for an individual $Z_{2}$ instanton is logarithmic divergent. When the textures are already present, the interaction between the spin disclinations in the $Z_{2}$ instantons becomes finite ranged due to the loss of the spin stiffness at a distance longer than $\xi$. However, the half vortices in the $Z_{2}$ instantons still interact with each other via a logarithmic potential when the phase remains coherent. So the quantum tunneling of the $Z_{2}$ instantons are absent in disordered nematic states in the weakly interacting limit.

The suppression of the $Z_{2}$ instantons caused by the half vortices also suggests that the $Z_{2}$ gauge fields, when coupled with a phase coherent BEC, be "frozen" in $1+1$ dimension in the weakly interacting limit. The $Z_{2}$ gauge field is effectively decoupled from the spin dynamics and the spin order-disorder transitions are driven by the spin waves in the $S^{2}$ sector, which is self-consistent with the assumption we made at the beginning of the section. On the other hand, when both the phase and the spin sectors are disordered, we expect the presence of the $Z_{2}$ instantons in $1+1$ dimension. A quantitative calculation of the multi-influence between the $\pi$ spin disclinations and the textures, especially the $\pi$ spin disclinations effect on the Haldane gap is still absent 70 .

\section{SDQCS UNDER THE INFLUENCE OF $Z_{2}$ FIELDS}

The influence of the $Z_{2}$ symmetries in symmetry unbroken states, say SDQCs, can be most conveniently examined in the lattice gauge field model discussed in section II. The action in Eq.23 represents an $X Y$ model and a Heisenberg model coupled with a $Z_{2}$ gauge field $\sigma_{r r^{\prime}}$. In the limit when $K$ goes to infinity, the $Z_{2}$ gauge fields are frozen and $\sigma_{r r^{\prime}}=1$; Eq.23 reduces to an $X Y$ model for the phase sector and a Heisenberg model for the spin sector. The spin dynamics in this case is identical to that of an NL $\sigma \mathrm{M}$, as discussed in details in an early section. The dimensionless coupling constants for the $X Y$ model and the NL $\sigma \mathrm{M}$ in optical lattices are

$$
f_{p}^{o . l .}=\sqrt{\frac{u}{J^{c}}}, f_{s}^{o . l .}=\sqrt{\frac{g}{J^{s}}}
$$

where $g$ and $u$ as defined in Eq.19 depend on the spin susceptibility and the compressibility. By carrying out calculations similar to those in section II, one obtains $u=4 \pi \rho\left(2 a_{2}+a_{0}\right) / 3 M N_{0}$ and $g=4 \pi \rho\left(a_{2}-a_{0}\right) / 3 M N_{0}$ in terms of scattering lengths $a_{0,2}$, the number density $\rho$ and the mass of atoms $M$.

In the weakly interacting limit where $f_{s}^{0 . l}$ is much less than unity, the rotation symmetry is broken in the ground state and nematic order occurs. This order leads to birefringence of a polarized light [37] and enhanced small angle light scatterings. However, when $f_{s}^{o . l}$ is much larger than unity, the interaction between Goldstone modes results in a spin disordered liquid. Similar to a resonant-valence- bond (RVB) liquid in Heisenberg antiferromagnetic systems, a spin disordered liquid is rotationally invariant and thus is free from birefringence.

When $J^{s, c}$ approaches zero, Eq.23 becomes identical to a pure $Z_{2}$ gauge theory, which is dual to an Ising model [72. There are two phases for a pure $Z_{2}$ gauge theory at the spatial dimension greater than one. When $K>K_{c}$, the Wilson loop integral (defined in the next section) decays exponentially as a function of an area enclosed by the Wilson loop, i.e. in an area law. When $K<K_{c}$, the Wilson loop integral decays exponentially as the function of the perimeter of the loop. In the former case, two $Z_{2}$ charges interact with a potential linearly proportional to the distance between charges, therefore are confined. This has a rather profound impact on the quantum numbers of quasiparticles which will be studied in some detail in section VII.

In general, the BECs characterized by Eq.23 exhibit extremely rich physics because of a coupling between the spin degree of freedom and $Z_{2}$ gauge fields. The influence of the $Z_{2}$ symmetry in symmetry broken states was discussed in the previous section and here we want to further explore this influence in some symmetry partially restored states. Particularly, we will be interested in fractionalization of topological excitations due to the $Z_{2}$ gauge fields.

\section{A. Fractionalization of integer vortices}

There are three types elementary topological defects in the BEC of ${ }^{23} \mathrm{Na}$ [2]: 
1)a half-vortex around which the phase of the condensate changes $\pi$;

2)a $\pi$ spin disclination around which $\mathbf{n}$ rotates by $180^{\circ}$; 3)a frustrated plaquette with $\prod_{\square} \sigma_{i j}=-1$, or a $Z_{2}$ vortex. A $Z_{2}$ vortex emits a string of $\sigma_{i j}=-1$ links terminated either at the boundary or at another $Z_{2}$ vortex.

In general, when the $U(1)$ symmetry or the $S^{2}$ symmetry is broken, an individual half vortex or a $\pi$-disclination or a $Z_{2}$ vortex is an energetic catastrophe because of a cut, running from the defect to infinity, along which the phase of the condensate has to change abruptly. As a result, the $Z_{2}$ vortices have to be confined and form bound states with either the half vortices or the $\pi$ disclinations in the phases where the $U(1)$ or $S^{2}$ and the $Z_{2}$ symmetries are broken. In a polar state studied before, where all three symmetries are broken, only composites of the half vortices and the $\pi$ spin disclinations superimposed with the $Z_{2}$ gauge vortices are free objects [37,42]. On the other hand, in the states where both the $U(1)$ and $S^{2}$ symmetry are unbroken, the $Z_{2}$ vortices are free excitations.

To demonstrate fractionalization of topological excitations in the $S D Q C$ explicitly, we consider a specific situation where the $U(1)$ symmetry is broken but the $S^{2}$ symmetry is restored. This happens in $1 \mathrm{~d}$ in a weakly interacting limit and also in higher dimensions. So the $\pi$ disclinations are always deconfined and are liberated. By integrating out $\mathbf{n}$ of the nematic spin liquid, one obtains an effective action for $\exp (i \chi)$,

$$
\mathcal{S}=-J_{X Y} \sum_{r r^{\prime}} \sigma_{r r^{\prime}} \cos \chi_{r r^{\prime}}-K_{Z_{2}} \sum_{\square} \prod_{\square} \sigma_{r r^{\prime}}
$$

with $J_{X Y}, K_{Z_{2}}$ being finite, renormalized by gapped spin fluctuations [42]. Eq.45 represents an $X Y$ model coupled with a $Z_{2}$ gauge field. A gauge model similar to Eq.45 was proposed for the classical $O(3)$ nematic orderdisorder phase transitions [73]. A similar model was also considered and investigated in the context of cuprate high-temperature superconductors. [46].

The BECs described by the reduced action in Eq.45 admit one ordered and two disordered phases. At large $K_{Z_{2}}$ limit, a nematic order-disorder phase transition takes place, with integer vortices condensed but half vortices gapped. The energy of half vortices per unit length is finite in the disordered phase. This state as discussed in section VII supports spin one excitations and is a fractionalized phase. At small $K_{Z_{2}}$ limit, another phase transition occurs, but with half vortices condensed: the energy of a half-vortex per unit length vanishes in the disordered phase. It is a confining phase where only spin two excitations exist. Along the $J_{X Y}=0$ axis, there is a transition between these two disordered phases, the fractionalized one and the confining phase, as a pure gauge field phase transition.

When $J_{X Y}$ is large, the $U(1)$ symmetry is broken. Unlike in one-component BECs where an elementary topo- logical excitation carries a unit circulation, an elementary excitation in the BEC with a hidden $Z_{2}$ symmetry is a half vortex. To see this, let us start with the limit where $K_{Z_{2}}$ is large but finite. The energy density of an isolated half vortex per unit length is

$$
E_{h . v .}=\frac{\rho \pi \hbar^{2}}{8 M}\left(\ln \frac{L}{a}+\frac{L}{a}\right),
$$

with the second term being the energy of a cut. ( $L$ is the size of the system in $x y$ plane). Moreover, two halfvortices are bound to form an elementary excitation with a unit "flux quantum".

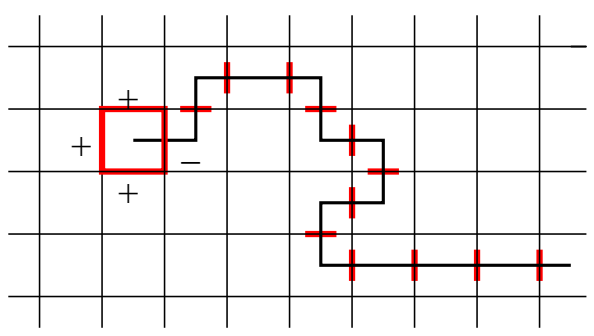

Fig.7 A $Z_{2}$ vortex emits a string of $\sigma_{i j}=-1$ links, represented by short-thick lines.

However, when a $Z_{2}$ vortex as shown in Fig.7 is inserted, all $\sigma_{r r^{\prime}}$ along the cut have negative signs and the energy catastrophe of a half vortex is removed. The energy of a $Z_{2}$ vortex is from the core where $\prod_{\square} \sigma_{i j}$ has a negative sign there. The finite energy cost of the gauge vortex is proportional to $K_{Z_{2}}$ (assuming $Z_{2}$ is deconfining). The total energy of the composite in the unit of $\rho \pi \hbar^{2} / 8 M$ is just $\ln L / a$, up to a logarithmic acuracy and is one fourth of the energy of an integer vortex. Therefore, an integer vortex will fractionalize into two half vortices and a half vortex becomes an elementary object. Let us emphasis this arises because of the discrete $Z_{2}$ gauge fields and doesn't happen in spinless BECs or an $X Y$ model.

\section{B. BECs of singlet pairs}

Upon taking into account other higher order processes, we have to include a hopping integral of singlet pairs as in Eq.23. The pair hopping integrals $J^{2 s, 2 p}$ are particularly important when $g$ is large and states with odd number of atoms in individual wells are energetically costly: they are not allowed to have $L=0$ state of a rotor due to parity constraint and have a higher rotational energy. It is energetically favorable to have even numbers of atoms on each well arranged into singlet combinations, which can be interpreted as binding of atoms into singlet pairs with a pair binding energy 


$$
E_{p}(Q)=2 E(Q+1)-E(Q+2)-E(Q)=g .
$$

In the limit when $g \rightarrow \infty$ a crystal phase is possible for even numbers of atoms per well and corresponds to a spin singlet insulator. The superfluid phase is a condensate of singlet pairs (SSC), in which tunneling of individual atoms between the wells is suppressed and only singlet pairs are delocalized. The origin of pairing in this case is not the attraction between individual bosons, but a singlet formation on the scale of individual wells. This reminisces the "attraction from repulsion" mechanism of electron pairing proposed by Chakravarty and Kivelson for high Tc cuprates, $C_{60}$, and polyacetylene [74. The basic idea of this type is also the foundation of Anderson's interlayer tunneling mechanism for the high$T_{c}$ cuprates 75$]$. In general, when a liquid is one particleincompressible but two-particle compressible due to either a spin gap as in our case, or more general due to infrared catastrophes, the two-particle tunneling between liquids is encouraged and long-range order of pairs can be established 76].

Formally speaking, a large pair-tunneling term reduces the $U(1)$ symmetry to the $Z_{2}$ symmetry; only $\phi=0$ or $\pi$ on the unit circle is relevant for the low energy physics. The planar spins in Eq.45 therefore have to be replaced by Ising spins $S_{r}= \pm 1$ and one obtains

$$
\mathcal{S}=-J_{I} \sum_{r r^{\prime}} \sigma_{r r^{\prime}} S_{r} \cdot S_{r^{\prime}}-K_{Z_{2}} \sum_{\square} \prod_{\square} \sigma_{r r^{\prime}} .
$$

The action in Eq.48 admits only two distinct phases, as pointed out by Fradkin and Shanker 77]. Along the $K_{Z_{2}}=\infty$ axis, a usual Ising order-disorder transition takes place. Along the $J_{I}=0$ axis, there will be a transition from the $Z_{2}$ confining phase to the $Z_{2}$ deconfining phase. But along the $K_{Z_{2}}=0$ axis, no transitions occur. So two symmetry broken but topologically distinct phases are allowed: a) an Ising ordered phase with $<S_{i}>\neq 0$ and confining $Z_{2}$ fields and b) an Ising disordered phase with $\left\langle S_{i}\right\rangle=0$ and deconfining $Z_{2}$ fields.

For BECs, the first phase corresponds to a conventional single atom condensate with $\langle\cos \chi>\neq 0$ and the latter one is an exotic singlet pair condensate where $<\cos \chi>=0$ but $<\cos 2 \chi>\neq 0$, first pointed out in 442,43. Topological excitations have distinct composition in these two symmetry broken states. The Ising ordered phase breaks the $Z_{2}$ and $U(1)$ symmetries. The half vortices and the $Z_{2}$ gauge vortices are confined. However, in the Ising disordered state, the $Z_{2}$ symmetry is restored while the $U(1)$ symmetry is still broken. The half-vortex and $Z_{2}$ vortex are liberated and are free excitations. The transition between these two states is of the Ising universality. The other interesting aspect of the singlet pair condensate is the a.c. Josephson effect. For the pair condensates,

$$
\frac{d\left(\chi_{1}-\chi_{2}\right)}{d t}=2\left(\mu_{1}-\mu_{2}\right)
$$

where $\chi_{1,2}$ and $\mu_{1,2}$ are the phases and chemical potentials of two BECs. Note that the factor 2 appears in the right hand side of the equation for the pair BEC. A more realistic proposal was given in 443].

\section{HIDDEN TOPOLOGICAL ORDER}

As emphasised through out this paper, the spinor BECs of spin one atoms have many fascinating properties associated with the unique topology of the spin order parameter and the entanglement of spin-phase degrees freedom. These interesting topological aspects result in composited topological excitations in symmetry broken states, rotationally invariant spinor BECs and fractionalization of topological excitations in symmetry restored states.

In this section, I will explore the conservation of topological charges. Particularly, I want to identify hidden topological order in spinor BECs. Furthermore, I generalize these ideas to the rotationally invariant spin liquids and show that topological order coexists with a shortrange spin correlation, as a result of the topological order from disorder phenomena. Classification of electron liquids using quantum orders was recently reviewed by Wen [78]. Similar ideas have been implemented for spin triplet superconducting liquids [47.

\section{A. Hidden topological order in Pontryagin fields in BECs}

As discussed at the beginning of the section IV, under the assumption that the $Z_{2}$-strings are gapped, one can introduce a connection field to characterize all configurations of $\mathbf{n}$ (see also [44,45]). The connection field is defined as

$$
\mathbf{A}(\mathbf{r})=\epsilon^{\alpha \beta} \mathbf{e}_{\alpha} \nabla \mathbf{e}_{\beta}
$$

here $\mathbf{e}_{\alpha}$ and $\mathbf{n}$ form a local triad; $\mathbf{e}_{\alpha} \cdot \mathbf{e}_{\beta}=\delta_{\alpha \beta}, \epsilon_{\alpha \beta} \mathbf{e}_{\alpha} \mathbf{e}_{\beta}=$ $2 \mathbf{n}$ and $\mathbf{e}_{\alpha} \cdot \mathbf{n}=0$. The field strength is a generalized gauge invariant Pontryagin density

$$
\mathbf{F}_{\mu \nu}=\epsilon^{\alpha \beta \gamma} \mathbf{n}_{\alpha} \partial_{\mu} \mathbf{n}_{\beta} \partial_{\nu} \mathbf{n}_{\gamma}
$$

In $(3+1) d, \mathbf{F}_{0 i}, i=x, y, x$ corresponds to an electric field and $\epsilon^{i j k} \mathbf{F}_{i j}=\mathbf{H}_{k}$ represents three components of a magnetic field; in $2 d, \mathbf{F}_{0 i}, i=x, y$ is an electric field with two components and the magnetic field has only the $z$ component $\mathbf{H}_{z}=\mathbf{F}_{x y}$. 


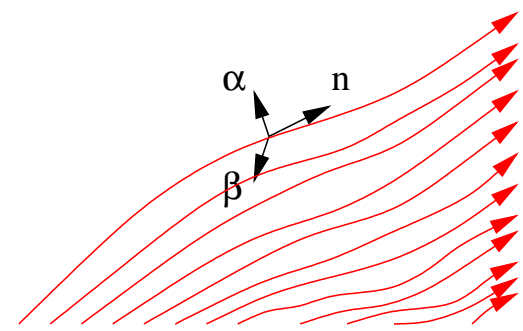

Fig. 8 The local triad defined at each point $\mathbf{r}$.

One will be interested in the fluctuations of a topological charge defined as

$$
C_{U(1)}(\{\mathbf{n}(\mathbf{r})\})=\frac{1}{4 \pi} \oint_{\mathbf{s}} \mathbf{d S H} \cdot \mathbf{e}_{\mathbf{n}},
$$

where the integral is carried over the boundary of a large volume $\mathcal{V}$ and $\mathbf{e}_{n}$ is a unit vector normal to the boundary. $C_{U(1)}$ counts the number of hedgehogs in the volume $\mathcal{V}$. When the topological charge is a conserved quantity, topological different configurations are well-defined distinguishable ones. Otherwise, topological distinct configurations are converted into each other by certain instantons.

\section{Topological order}

I am going to show that at large $\mathcal{V}, t$ limit,

$$
\lim _{t, \mathcal{V} \rightarrow \infty}<\left[C_{U(1)}(\{\mathbf{n}(\mathbf{r})\}, t)-C_{U(1)}(\{\mathbf{n}(\mathbf{r})\}, 0)\right]^{2}>=0
$$

in both spin ordered and spin weakly disordered BECs, as results of topological long-range order. The topological order occurs when hedgehogs are confined, either because of the condensation of atoms or an order from disorder mechanism. However, in the strongly disordered limit,

$$
\lim _{t, \mathcal{V} \rightarrow \infty}<\left[C_{U(1)}(\{\mathbf{n}(\mathbf{r})\}, t)-C_{U(1)}(\{\mathbf{n}(\mathbf{r})\}, 0)\right]^{2}>=\frac{t \mathcal{V}}{\tau_{m}}
$$

where $\tau_{m}$ is finite depending on the tunneling rate of magnetic monopoles(see below). The averages in Eqs. 53,54 are taken over the many-spin wave function $\Psi(\{\mathbf{n}\})$. In $2 \mathrm{~d}$, we define

$$
C_{U(1)}(\{\mathbf{n}(\mathbf{r})\})=\frac{1}{4 \pi} \int d x d y \mathbf{H}_{z},
$$

the total number of Skyrmions living in the $2 \mathrm{~d}$ space and Eqs.53,54 hold in the spin ordered and spin disordered BECs respectively $(\mathcal{V}$ is replaced by an area $\mathcal{S})$.
More formally, we can introduce an order parameter of the Wilson loop type to characterize the hidden topological order. The Wilson-loop integral defined as

$$
W_{U(1)}=\left\langle\mathcal{P} \exp \left(i \oint_{\mathcal{C}} \mathbf{A} \cdot d \mathbf{r}\right)\right\rangle
$$

has different asymtotical behaviors in the large loop limit in the presence or absence of the topological order. When the topological charge $C_{U(1)}$ is conserved, the instantons which connect topological different configurations are forbidden; the exponent in the Wilson loop integral is a linear function of the perimeter of the loop. On the other hand when $C_{U(1)}$ is not conserved, the instantons are allowed and one can confirm the exponent of the Wilson loop integral is a linear function of the area enclosed by the loop. Though the Wilson loop integral was originally introduced for pure gauge fields, here we employ it for the characterization of the topological order in the spinor BEC where gauge fields are coupled with coherent matter fields.

The topological conservation law has an important consequence on collective excitation spectra in spin disordered BECs. One can show that when the instantons are present, the connection field has a massive longitudinal component (with a mass $m_{c}$ )

$$
\left\langle\mathbf{H}_{i} \mathbf{H}_{j}\right\rangle=\left(\delta_{i j}-\frac{\mathbf{k}_{i} \mathbf{k}_{j}}{k^{2}}\right)+\frac{\mathbf{k}_{i} \mathbf{k}_{j}}{k^{2}} \frac{m_{c}^{2}}{k^{2}+m_{c}^{2}} .
$$

And when the topological charge is conserved, the connection fields have only transverse components,

$$
\left\langle\mathbf{H}_{i} \mathbf{H}_{j}\right\rangle=\left(\delta_{i j}-\frac{\mathbf{k}_{i} \mathbf{k}_{j}}{\mathbf{k}^{2}}\right) .
$$

The topological rigidity can be introduced as

$$
\rho_{t . o .}=\frac{\partial^{2} E\left(\mathbf{F}_{0 j}\right)}{\partial \mathbf{F}_{0 j}^{2}} .
$$

When topological order is present, $\rho_{t . o} \neq \neq 0$; otherwise, $\rho_{\text {t.o. }}$ vanishes, indicating screening of a small connection field (electric component).

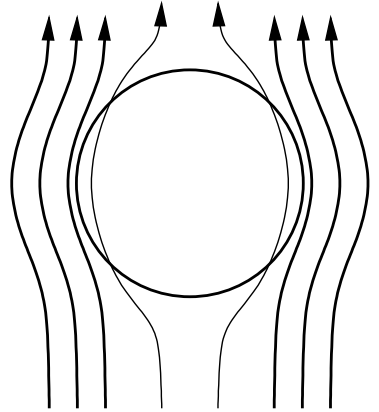

(a)

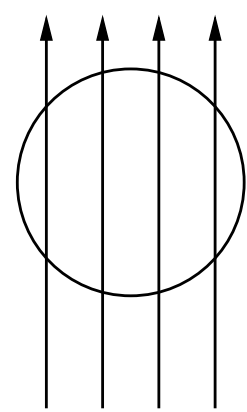

(b)
Fig. 9 The responses to an external connection field in the absence a) or presence b) of topological order. 


\section{Topological order and the spin stiffness}

To study the conservation of the topological charges, we first obtain the equation of motion for $\mathbf{H}_{k}$ using Eqs.8, 10

$$
\frac{\partial \mathbf{H}_{k}}{\partial t}=\epsilon_{i j k} 8 c_{2} \mathbf{n} \cdot \partial_{j} \mathbf{n l} \cdot \partial_{i} \mathbf{n}
$$

Eq.60 demonstrates that the topological charge is conserved if singular space-time events are not allowed so that the product $\mathbf{n} \cdot \partial \mathbf{n}$ in the right hand side of the equation vanishes identically.

And the topological charges fluctuate only when singular space-time events are permitted in the ground state. This is rather explicit in $2 d$. First, we notice that in space $\mathbf{x}=(t, \mathbf{r}), \mathbf{H}_{\eta}=\epsilon^{\eta \mu \nu} \mathbf{F}_{\mu \nu}$ is the solution of the Gauss equation

$$
\partial_{\eta} \mathbf{H}_{\eta}(\mathbf{x})=\sum_{m} Q_{m} \delta\left(\mathbf{x}-\mathbf{x}^{m}\right)
$$

in the presence of space-time monopoles $\left\{\mathbf{x}^{m}\right\}$ in $(2+1) d$ Euclidean space.

The space-time monopoles represent the instantons, which essentially connect a trivial vacuum to a Skyrmion configuration. In Euclidean space, a spacetime monopole located at $x=\left(t_{0}, \mathbf{r}_{0}\right)$ terminates a Skyrmion centered at $\mathbf{r}_{0}$ at time $t_{0}$. There are two important aspects in Eq.61. The first is that the topological density is conserved in the absence of the spacetime monopoles in $(2+1) D$. Second, each monopole event causes a change in the topological charge $C_{U(1)}$ by one unit; indeed, following Eqs.55,61,

$$
\frac{\partial C_{U(1)}(\tau)}{\partial \tau}=\sum Q_{m} \delta\left(\tau-\tau_{m}\right)
$$

where the surface contribution has been neglected since we will be interested in the leading contribution to $C_{U(1)}$ per unit square.

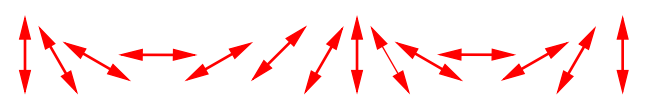

(a)

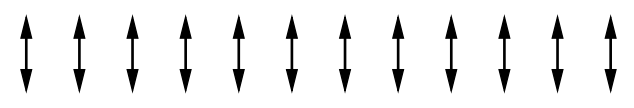

(b)

Fig.10 The spin configurations at a) $t=+\infty$, and b) $t=-\infty$ boundaries of a space-time monopole event; the orientation of a spin is indicated by an arrow.

The probability of finding deconfined space-time monopoles depends on the energy of the skyrmions of $C_{U(1)}=1$ with respect to a $C_{U(1)}=0$ configuration. In polar coordinates $\left(r, \phi_{P}\right)$, a static Skyrmion is a configuration with

$$
\mathbf{n}\left(r, \phi_{P}\right)=\left(\sin \theta(r) \cos \phi_{P}, \sin \theta(r) \sin \phi_{P}, \cos (r)\right) ;
$$

$\theta(r)$ varies from 0 at $r=0$ to $\pi$ at $r \gg \xi$, with $\xi$ an arbitrary parameter. For spin ordered BECs where the spin Josephson effects should be observed, the energy of a Skyrmion is proportional to $8 \pi \hbar^{2} \rho / 2 M$ and is scale invariant, as reflected in Eq.36 as well. Without lossing generality we consider a Skyrmion of a given size $\xi[79]$. The connection field is concentrated in a region of the size $\xi$,

$$
\mathbf{H}_{z}=\frac{1}{r} \sin \theta(r) \frac{\partial \theta(r)}{\partial r}
$$

and gets screened at a length scale larger than $\xi$. In spin ordered BECs, a Skyrmion is nondegenerate with respect to a trivial vacuum. As one will see, this leads to the confinement of space-time monopoles.

To illustrate the point of the confinement, one considers $C_{U(1)}$ as a function of time measured in some discrete units and find the following binary representation for monopoles.

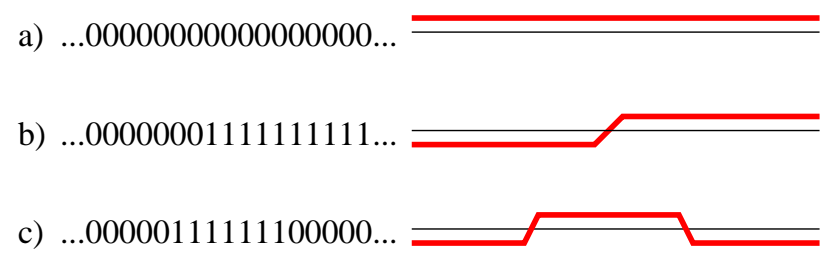

Fig.11 In a binary representation, monopole-like instantons are represented by kinks living on a string with $C_{U(1)}$, the topological charge as the order parameter.

Bits labeled as 0,1 as shown in Fig.11 represent the topological charge read out at each moment; a 1-bit corresponds to a Skyrmion while a 0 -bit is for a $C_{U(1)}=0$ configuration. The a) string represents a trivial vacuum where $C_{U(1)}=0$ at all time. In the second binary string b), a 1-string stands for any scale invariant Skyrmion created at the time $t_{0}$ defined by the domain wall separating the 0 -string and 1-string. So a domain wall in our binary representation stands for a monopole $Q_{m}=1$, terminating a Skyrmion configuration $\left(C_{U(1)}=1\right)$ at the interface and increasing $C_{U(1)}$ by one unit. A domain wall-anti domain wall pair in the third line c) corresponds to a monopole-antimonopole pair with $Q_{m}= \pm 1$; a 1-string here, or a Skyrmion, is terminated at a monopole at one end $t_{1}$ and at an antimonopole at the other end $t_{2}$.

Since the energy of a Skyrmion is higher than that of a trivial configuration, the "energy" of a 1-bit is positive with respect to a 0-bit. The energy of a domain wall in Fig. 11 which is the number of 1-bits in the structure, defines the action of the monopole event and is proportional 
to the length of the 1-string. The action of an isolated space-time monopole event, or the creation of a Skyrmion therefore is proportional $L_{t} \hbar \rho / 2 M$, being infinity. ( $L_{t}$ is the perimeter along a temporal direction.) The action of having monopole-anti-monopole pairs is proportional to $\left(t_{1}-t_{2}\right)$, the time interval between the creation and annihilation of the skyrmions, thus the monopoles are confined. For this reason, the right hand side of Eq.62 vanishes and topological order as shown in Eq.53 is established.

The conclusion arrived so far can be extended to $3 \mathrm{~d}$ straightforwardly. The change of the topological density is caused by the quantum nucleation of monopoles instead of Skyrmions. In the binary representation in Fig.11, a 1-bit stands for a static monopole and a 0-bit for a trivial configuration. A domain-wall represents termination of a static monopole at certain time. Since the energy of a monopole is proportional to the system size $L$ in a spin ordered BEC, the monopole is nondegenerate with respect to the trivial vacuum. Following the same argument carried out in $2 \mathrm{~d}$, the action to have the monopole nucleated is proportional to $L L_{t} \hbar \rho / 2 M$, and is infinity. This result has an interest impact on the monopole confinement. The interaction between a monopole and an antimonopole has to be linear in terms of distance between them. The action of a monopole pair nucleated at $t_{1}$ and annihilated at $t_{2}$ with the largest spatial separation as $\left|r_{1}-r_{2}\right|$ is proportional to $\left|r_{1}-r_{2}\right|\left(t_{1}-t_{2}\right)$ and the monopoles are confined due to the spin stiffness. Therefore the right hand side of Eq.62 has to be zero because of the confinement, and the topological charge $C_{U(1)}$ is a conserved quantity. We once again arrive at Eq.53 in the spin ordered BEC.

\section{Order from disorder}

The situation in the disordered limit is more delicate and depends on dimensionalities. In the spin disordered $\mathrm{BEC}$, the spin stiffness is renormalized to zero and the spin fluctuations are gapped. However, the spin fluctuations do depend on the background connection fields. This is explicit in Eq. B3 in the appendix B. Upon integrating over spin wave excitations, based on a general consideration of the gauge invariance and the parity invariance, we conclude the $\mathrm{NL} \sigma \mathrm{M}$ should be reduced to

$$
\mathcal{S}_{s}\left(\mathbf{F}_{\mu \nu}\right)=\frac{1}{2 g_{1}} \int d^{d} \mathbf{r} d \tau \mathbf{F}_{\mu \nu} \mathbf{F}_{\mu \nu}+\ldots
$$

$g_{1}\left(\Delta_{s}\right)$ is a function of $\Delta_{s}$, the spin gap measured in units of $\sqrt{c_{2} \rho^{5 / 3} / 2 M^{2}}$ [80]. And in a large- $\mathrm{N}$ approximation,

$$
\frac{1}{g_{1}} \propto\left\{\begin{array}{cc}
\ln \Delta_{s}^{-1}, & 3 d \\
\Delta_{s}^{-1}, & 2 d
\end{array}\right.
$$

We will analysis the topological order based on $\mathcal{S}_{s}$. The energy of a $C_{U(1)}$-configuration is $\alpha(L) C_{U(1)}^{2}$ if the quantum tunneling is neglected, with $\alpha(L)$ being a function of the system size.

In the absence of the spin stiffness, the energy of a Skyrmion or a monopole is determined by its interaction with the spin fluctuations. This contribution to the energy of a skyrmion excitation in a Neel ordered antiferromagnet was previously considered by Auerbach et al. [81]. The action of topological instantons was estimated by Murthy and Sachdev, Read for quantum disordered antiferromagnetic spin liquids [32,83].

In $2 \mathrm{~d}$, with the induced interactions, the Skyrmion energy is no longer scale invariant and is minimized at the size $\xi=\infty$. The connection field of a Skyrmion spreads over the whole $2 \mathrm{~d}$ sheet. The energy of a Skyrmion scales as $L^{-2}$ and vanishes as the system size $L$ goes to infinity. This implies that the skyrmion configurations become degenerate with a trivial vacuum and the monopoles are deconfined. This mechanism of deconfinement resembles the liberation of fractionalized quasiparticles in one dimension polymers 84. In one dimensional polyacetylene, the ground state has a two-fold degeneracy because of the Peierls instability and the domain wall solitons become free excitations.

Unfortunately, when the instantons are liberated and the topological charge $C_{U(1)}$ is not conserved, the energy of a configuration $C_{U(1)}$ is ill-defined. A more serious consideration involves the evaluation of the action of monopole-like instantons. In $(2+1) d$, the partition function of a monopole configuration $\left\{\mathbf{x}^{m}\right\}$ represents pointlike "charges" interacting via long-range Coulomb interactions. The result suggests that the skyrmions always condense in the spin disordered BEC and the topological charge $C_{U(1)}$ is not a conserved quantity. It is important to realize that positive and negative Skyrmions can be physically and homotopically distinguished because of the coherence of the BEC, unlike the situation in a classical nematic liquid crystal.

By moving a Skyrmion around a $\pi$-disclination, the condensate acquires a $\pi$-phase with respect to the original one. The $\pi$-phase difference, which can manifest itself in a Josephson type of effect, is one of the signatures left behind by the positive or negative Skyrmions. One can also distinguish \pm Skyrmions by looking at the local connection field. In a positive Skyrmion configuration, a spin- $\frac{1}{2}$ collective excitation, which carries a half charge with respect to the connection fields, experiences a connection field of an opposite sign compared to that of a negative Skyrmion 42]. This is similar to the situation of a point defect discussed in section III.

So one is able to show that the change of $C_{U(1)}, \partial_{t} C$, has a short-range temporal correlation because of the instanton effects, 


$$
<\partial_{t} C_{U(1)}(t) \partial_{t} C_{U(1)}(0)>=\exp \left(-\frac{t}{\tau_{m}}\right) .
$$

And $\tau_{m}^{-1}$ is proportional to $L^{2} \xi^{-2} \tau^{-1} \exp \left(-S_{0}\right)$. Immediately, one recognizes that Eq.67 simply indicates a random walk of $C_{U(1)}$ as a function of time in spin disordered 2d BECs. Therefore, one concludes Eq 54 holds.

But in $3 d$ weakly disordered BECs where the stiffness has vanished, a static monopole with $\mathbf{H}_{k}=\mathbf{r} /|\mathbf{r}|^{3}$ still carries a finite energy because of the spin fluctuations induced interactions. In $3 d$, as illustrated in $\mathcal{S}_{s}\left(\mathbf{F}_{\mu \nu}\right)$, the spin fluctuations discriminate topologically different configurations. Particularly, the fluctuations are strongest in a $C_{U(1)}=0$ configuration so that the energy is lowest in the topologically trivial configuration. The energy of a monopole $C_{U(1)}=1$ is finite with respect to the $C_{U(1)}=0$ configuration.

The situation differs from the $2 \mathrm{~d}$ spin disordered limit where the energy of a topologically nontrivial configuration with $C_{U(1)}=1$ is the same as that of $C_{U(1)}=0$ one. For a monopole, each spherical shell of radius $R$ can be viewed as a Skyrmion squeezed into a $2 d$ sheet of size $R$. While the energy of a shell with a large radius is unsubstantial, the smaller shells, which correspond to textures of finite sizes and have finite energies, dominate the energy of a monopole in the spin disordered limit. Because of the singular structure of the monopole, the coupling between a monopole and the spin fluctuations lifts the degeneracy between the monopole configuration and a trivial vacuum in the absence of the spin stiffness. It also appears to us that in $3 d$ the spin fluctuations should order the topologically different configurations energetically, independent of the specific form of $\mathcal{S}_{s}\left(\mathbf{F}_{\mu \nu}\right)$ we introduced. The energy of a monopole here is inversely proportional to $g_{1}$, i.e. $\left(E_{o} E_{T}\right)^{1 / 2} \ln \Delta_{s}^{-1}$. The action of having a monopole, which leads to a change in $C_{m}$ by one unit, is proportional to $L_{t}$ and is infinite for this reason.

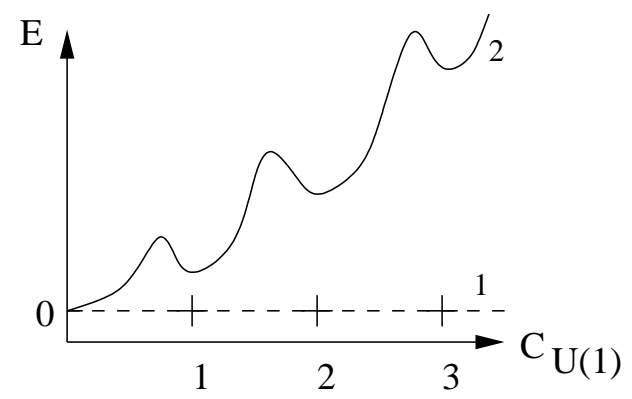

Fig.12 The energy of the monopoles of charge $C_{U(1)}$ in the absence of spin stiffness. Curve 1 is the result in a mean field approximation and curve 2 schematically represents the interaction energy between a monopole and spin fluctuations in a finite system.

The monopoles remain confined even after the $S^{2}$ - rotation symmetry is restored, with the action of having a pair of monopoles proportional to $\left(t_{1}-t_{2}\right)$. Only the pair production which conserves $C_{U(1)}$ is allowed. The topological order thus coexists with a short-range spin correlation in $3 d$. This can be considered as a case of order from disorder phenomena. Though topological ordering occurs in symmetry unbroken states, it mimics the conventional order from disorder phenomena as far as the role of the fluctuations is concerned 85,86,87. For instance, the spectra of the spin fluctuations are affected by a topologically nontrivial back ground in our case and by the symmetry breaking in 85, 86, 87. Topological order is established as a result of the confinement; the confinement of the monopoles in this case is driven purely by the spin fluctuations, instead of the spin stiffness in spin ordered BECs. However, by increasing the spin disorder further, the degeneracy between $C_{U(1)}=1$ and $C_{U(1)}=0$ could be established. This signifies the deconfinement of the monopoles and the breakdown of the topological order. $C_{U(1)}$ again will have a Brownian motion as the time evolves, implied by Eq.67.

\section{B. Topological order in $Z_{2}$ fields}

A discussion of the hidden topological order of the $Z_{2}$ fields can be carried out parallel to the previous discussion of $U(1)$ fields. For simplicity, I present the result in $2 d$ case, though a generalization is possible. It is particularly convenient to look at the Wilson loop integral of the $Z_{2}$ fields, which takes a form

$$
\mathcal{W}_{Z_{2}}=\left\langle\prod_{\mathcal{C}} \sigma_{r r^{\prime}}\right\rangle
$$

with the product carried out along the loop $\mathcal{C}$. Once again, the exponent is a linear function of the perimeter when the $Z_{2}$ vortices are gapped and is proportional to the area when the $Z_{2}$ vortices condense. Consequently, the topological charge $C_{Z_{2}}$ in $2 d$ defined as

$$
C_{Z_{2}}\left(\left\{\sigma_{i j}\right\}\right)=\prod_{\mathcal{C}} \sigma_{i j}
$$

is a conserved quantity when the $Z_{2}$ vortices are forbidden dynamically and is nonconserved when the $Z_{2}$ vortices are allowed.

In the presence of topological order

$$
\lim _{t, \mathcal{V} \rightarrow \infty}<\left[C_{Z_{2}}\left(\left\{\sigma_{i j}\right\}, t\right)-C_{Z_{2}}\left(\left\{\sigma_{i j}\right\}, 0\right)\right]^{2}>=0,
$$

and in a topologically disordered phase

$$
\lim _{t, \mathcal{V} \rightarrow \infty}<\left[C_{Z_{2}}\left(\left\{\sigma_{i j}\right\}, t\right)-C_{Z_{2}}\left(\left\{\sigma_{i j}\right\}, 0\right)\right]^{2}>=\frac{t \mathcal{V}}{\tau_{m}} .
$$

In BECs where either the phase or spin is ordered, the $Z_{2}$ vortices are bound with either the half-vortices 
or the $\pi$-spin disclinations. The energy of the composite and the $Z_{2}$ vortices is determined by the stiffness, of the phase or spin. The energy of the $Z_{2}$ vortices is therefor logarithmic divergent. In the same spirit of the previous section, we conclude that the $Z_{2}$ vortices are forbidden and confined. The $Z_{2}$ charge $C_{Z_{2}}$ is a conserved quantity in the spin or phase ordered BECs, as a result of the topological confinement. Following this, the Wilson loop decays in a fashion of a perimeter law.

It is perhaps more interesting to look at the possible topological order in spin disordered BECs. The spin fluctuations discriminate the configurations with different $Z_{2}$ charges and the fluctuations are maximized in a trivial configuration. Therefore, the energy of a $Z_{2}$ charge $C_{Z_{2}}=1$ is higher than a trivial one of $C_{Z_{2}}=0$, though the spin stiffness vanishes.

Indeed, because of a coupling between the $Z_{2}$ fields and the spin fluctuations, an effective interaction of the $Z_{2}$ fields is induced upon an integration of spin fluctuations,

$$
\mathcal{S}_{i n}=-\frac{1}{2 g_{2}} \sum_{\square} \prod_{\square} \sigma_{r r^{\prime}}
$$

$g_{2}$ is a function of the spin gap developed in disordered limit 42]. Eq.72 once more illustrates a general idea of the topological order from disorder. A $Z_{2}$ vortex $\prod_{\square} \sigma_{i j}$ does acquire an energy, by interacting with the spin fluctuations. The energy of a $Z_{2}$ vortex in spin disordered BECs depends on the spin gap; it is finite but enormous in the spin weakly disordered limit in $2 d$, reflecting an energetic difficulty to have $Z_{2}$ vortices due to the weakly gapped spin fluctuations. The rest of discussions can be carried out in a binary representation. The action of an 1-string, representing termination of a $Z_{2}$ vortex is infinity. The instantons connecting configurations with different $Z_{2}$ charges are suppressed and $C_{Z_{2}}$ is conserved. Topological order can be established by the spin fluctuations.

\section{SYMMETRY RESTORING IN FINITE SIZE SPINOR BECS}

Since the experiment 30] was done in BECs of a few millions ${ }^{23} \mathrm{Na}$ atoms, it is also particularly interesting to consider the symmetry restoring due to a finite size effect. One takes a weakly interacting limit where quantum fluctuations of finite wave lengths are negligible. In a zero mode approximation, one can neglect the spatial fluctuations of $\mathbf{n}$ and obtain from Eq. 36

$$
\mathcal{C}_{\eta}(\mathbf{q}=0, t)-\mathcal{C}_{\eta}(\mathbf{q}=0,0)=-\frac{4 t c_{2} \rho \hbar^{2}}{N} \int d x \frac{\sin ^{2} x}{x^{2}}
$$

which is valid when the result is less than unity. $\mathcal{C}_{\eta}(\mathbf{q}=$ $0,0)$ should be considered of order unity. Formally speaking, the extrapolation of the above result to $t \rightarrow \infty$ shows that the correlator diverges as $t$ goes to infinity. This implies that $\mathbf{n}(t)$, at $t \sim N / c_{2} \rho$ (set by the two-body scattering length), becomes uncorrelated with the original orientation of $\mathbf{n}(0)$. At longer time scales, $\mathbf{n}$ precesses an extremely slow quantum diffusive motion on the unit sphere. That is to say, the nematic order parameter can rotate freely in the parameter space, as seen from $\mathcal{C}_{\eta}(0,0)$ as well and restore the broken rotation symmetry when $N$ is finite.

Following Eq.15, in the $0 D$ BEC the energy gap of the low lying excitations scales of $1 / N$ at large $N$ limit. In the ground state, $\langle\mathbf{n}\rangle=0$ because of the rotational invariance. Consider a wave packet with $\mathbf{n}$ confined within a region centered at $\mathbf{n}_{0}=\mathbf{e}_{z}$. The spread $\sqrt{\left\langle\delta^{2} \mathbf{n}>_{0}\right.}$ on the unit sphere at $t=0$ is very small. The spread $<\delta^{2} \mathbf{n}>$ is defined as the expectation value of $\mathbf{n}_{x}^{2}+\mathbf{n}_{y}^{2}$. In spherical polar coordinates $(\theta, \phi)$ defined on a unit sphere, $\mathbf{L}$ is a differential operator in terms of $(\theta, \phi)$ and the eigenstates of $\mathbf{L}^{2}$ are spherical harmonics $Y_{l, m}(\theta, \phi)$. The corresponding wave packet can be constructed as

$$
\begin{aligned}
& \Psi(\theta, \phi, t)=\frac{1}{B} \sum_{l=2 n} \exp \left(-\frac{l^{2}}{4 \sigma}-i t \frac{l(l+1) c_{2} \rho}{2 N}\right) Y_{l, 0}(\theta, \phi) \\
& B=\sum_{l} \exp \left(-\frac{l^{2}}{2 \sigma}\right),<\delta^{2} \mathbf{n}>_{0}=\frac{A_{0}}{\sigma}
\end{aligned}
$$

where constant $A_{0}$ is estimated in the Appendix C. $Y_{l, 0}(\theta, \phi)$ are spheric harmonics. Only states with even number $l$ are present in the wave packet(we assume $N$ is an even number at the moment). For this packet, $\left\langle\mathbf{n}_{x}\right\rangle=\left\langle\mathbf{n}_{y}\right\rangle=0$ and $\left\langle\mathbf{n}_{z}\right\rangle \approx 1$ when $\sigma \gg 1$ but $\sigma / N$ is vanishingly small.

The energy and the spread in $\mathbf{L}$ can be estimated as

$$
\Delta E=\frac{2 A_{0} \sigma c_{2} \rho}{N},<\delta^{2} \mathbf{L}>=4 A_{0} \sigma
$$

according to the derivation in the Appendix C. Therefore, an excited state where $\mathbf{n}$ is well defined on the unit sphere up to a spread $\sqrt{<\delta^{2} \mathbf{n}>_{0}}$ has an infinitesimal energy cost when $N$ approaches infinity.

The wave packet constructed in Eq.74, being energetically extremely close to the singlet ground state, indicates that the singlet is very unstable with respect to external magnetic fields. A field of the order $o(1 / N)$ will stablize the symmetry broken state in Eq.74. However, when one deals with BEC of a small number of atoms, the level spacing in the excitation spectrum gets bigger. The system becomes perturbative with respective to an external magnetic field, as far as the disturbance is less than the energy gap in the problem. So the claimed ultrasensitivity is simply a consequence of nearly degenerate low lying excitations in the large $N$ limit. Moreover, this ultrasen- 
sitivity is absent in the spin disordered states where a many body spin gap is opened up.

According to the equation of motion in Eq.11, as the time evolves, because of the two-body scatterings with $c_{2}>0, \mathbf{n}(t)$ gradually precesses along the finite $\delta \mathbf{L}$. Indeed, the spread of $\mathbf{n}$ at a time $t$ becomes

$$
<(\delta \mathbf{n})^{2}>_{t}=<(\delta \mathbf{n})^{2}>_{0}+4 A_{0} \sigma\left(t \frac{c_{2} \rho}{N}\right)^{2},
$$

which is valid at $t<\tau_{c}=\sigma / A_{0} \Delta E$. In an opposite limit the spread is of order unity.

Following Eq.76, at a time of order $1 / \Delta E, \sqrt{\left\langle\delta^{2} \mathbf{n}\right\rangle_{t}}$ exceeds the initial spread $\sqrt{<\delta^{2} \mathbf{n}>_{0}}$. At a longer time $\tau_{c}, \mathbf{n}$ starts to explore the whole unit sphere $S^{2}$ and the rotation symmetry is restored quantum mechanically in the presence of spin-dependent scatterings(see Fig.13). A discussion is present in appendix D when an external field is applied.

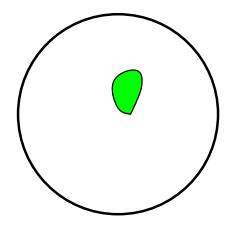

(a)

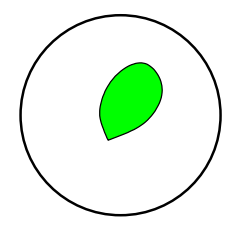

(b)

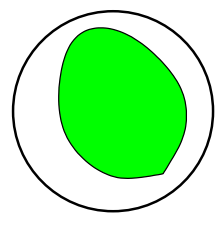

(c)
Fig.13 The symmetry restoring caused by the spindependent interactions $\left(c_{2}>0\right)$. Schematically shown are the spreads of $\mathbf{n}$ on a unit sphere at $t=0$ (a), $1 / \Delta E$ (b), $1 / \Delta E \times 1 / \sqrt{<\delta^{2} \mathbf{n}>_{0}}(\mathrm{c})$.

Eq.76 shows how a symmetry broken state in finite size BECs of ${ }^{23} \mathrm{Na}$ becomes a rotationally invariant state at an asymptotically long time. For ${ }^{23} \mathrm{Na}$ atoms interacting via a two-body interaction with $c_{2}>0$, it was argued in Ref. 32 that in a zero mode approximation the atoms should condensate at $|1, m=0\rangle$, with the rotation symmetry broken. On the other hand, based on an analogy to the nonlinear wave-mixing theory, it was shown in Ref. [34] that the exact ground state should be a spin singlet with the rotation symmetry preserved. The symmetry restoring in a finite size system as discussed above explains the apparent inconsistency between these previous theoretical works.

Given the number of particles as $6 \times 10^{6}$ and $\hbar^{2} / I_{0} \sim$ $100 \mathrm{~Hz}(500 \mathrm{nK})$, the typical time scale for this to take place is $t_{R}=N I_{0} / \hbar^{2} \sim 1$ day. This time scale is much longer than the life time of the trap [7]. Therefore the features associated with the singlet wave function proposed in Ref. 34] can not be observed under that particular experiment condition. However, for a smaller cloud with $10^{3-4}$ atoms, the symmetry restoring can take place within 10-100secs, before the recombination takes place. In this case, the ground state should be characterized by a singlet wave function, instead of a saddle point solution.
Eq.76 also imposes restrictions on the measurement of a symmetry broken state. An measurement of $\mathbf{n}$ in BECs of ${ }^{23} \mathrm{Na}$ discussed here excites the ground state to an excited state where $\mathbf{n}$ has a finite spread on $S^{2}$; the energy of this state is infinitesimal small in the thermal dynamical limit. The time scale for $\mathbf{n}$ to relax, or to restore the broken symmetry is determined by the two-body spin dependent scattering lengths and the number of atoms in BECs following Eq.76. Experimentally, the relevance of the singlet ground state or the symmetry broken state depends on the life time of the metastable gas or the interval between measurements. Any measurement taken at a time scale longer than $\sigma / A_{0} \Delta E$ should reveal the symmetry restoring because of zero point motions of $\mathbf{n}$. Possible measurements, including measurements of birefringence and small angle light scatterings, to detect orders in $\mathbf{n}$ in the weakly interacting limit were discussed in 37 .

It is worth emphasising that by contrast to a classical nematic liquid crystal where $\mathbf{n}$ is pinned along the external field and all the Goldstone modes are gapped, $\mathbf{n}$ can still freely rotate in a plane perpendicular to a weak magnetic field in the quantum spin nematic state under consideration. So the singularity in small angle light scattering survives an external magnetic field; only the amplitude is reduced by a factor of one-half, when an external field is applied. In a nematic disordered phase, the singularity gets smeared at $q \sim \xi^{-1}$ ( $\xi$ is the correlation length), which can be used to study a possible phase transition.

\section{DISCUSSION}

\section{A. Relation to an HAFS}

Though the NL $\sigma \mathrm{M}$ description of the quantum spin nematic states bears some similarities with that of the Heisenberg antiferromagnetic spin system(HAFS), there are at least two differences between the present problem and the Heisenberg problem. First, for the NL $\sigma \mathrm{M}$ of the HAFS, the ground state is invariant under a rotation on a sphere $S^{2}$; the order parameter space is a unit sphere $S^{2}$. $\pi_{1}\left(S^{2}\right)$ is homotopic to zero and the linear singularity is absent in this system. $\pi_{2}\left(S^{2}\right)=Z$ and the corresponding point defects are usual hedgehogs with a winding number $N$ distinguishable from $-N$. Overall, the topological defects in the Heisenberg spin system are qualitatively different from what we have in the QSNS described in section III.

However, when a weak magnetic field is applied, $\mathbf{n}$ in the NL $\sigma \mathrm{M}$ of the HFAS lives on a unit circle $S^{1}$ and $\pi_{1}\left(S^{1}\right)=Z$ is an integer group. The linear defects are integer vortices. Consequently, in $2 d$ in the presence of an external field, the interaction between topological linear 
defects in the QSNS at an asymptotically large distance decays in a same fashion as that in the HAFS. We expect that in this specific limit, the finite temperature phase transitions are of the same universality classes. In addition, $\pi$-spin disclinations superimposed with half vortices interact with each other via one half of the interaction strength between two integer vortices. Because of the reduced interaction strength between half vortices in the QSNS at $H \neq 0$, the critical temperature is lower than that of the HAFS.

It should be noticed that though the spin-dependent scatterings lift the degeneracy between different spin configurations and result in a spin nematic state when $c_{2}>0$, the critical temperature is determined by the density, i.e. $T_{c} \sim \hbar^{2} \rho^{2 / 3} / M$ which is independent of $c_{2}$ in the leading order approximation. When the zero point motions of $\mathbf{n}$ in weakly interacting BECs are taken into account, $T_{c}$ is shifted downward by an amount $o(f)$ proportional to $c_{2}$.

Second, in the Heisenberg antiferromagnetic system with a underlying spin $S$, both the zero point kinetic energy $(J S)$ and the potential energy $\left(J S^{2}\right)$ depend on the exchange integrals and the corresponding $f$, the dimensionless coupling constant in the NL $\sigma \mathrm{M}$, is inversely proportional to the spin $S$ only. This makes observing zero temperature order-disorder phase transitions extremely difficult. In the current situation, the potential energy is independent of the interaction while the zero point kinetic energy is proportional to the scattering length. $f$ is determined by the density and the scattering length instead of spins of Bosons and is a continuous variable in the QSNS.

\section{B. Classical nematic liquids}

The classical nematic order-disordered transitions were studied a few years ago based on a lattice gauge theory [73. The inversion symmetry of a nematic director is incorporated as a $Z_{2}$ gauge invariance in a lattice model,

$$
\frac{\mathcal{H}}{\| \mathcal{T}}=-J \sum_{r r^{\prime}} \sigma_{r r^{\prime}} \mathbf{n}_{r} \cdot \mathbf{n}_{r^{\prime}}-K \sum_{\square} \prod_{\square} \sigma_{r r^{\prime}}
$$

where $\sigma_{r r^{\prime}}= \pm 1$ are introduced as the $Z_{2}$ gauge fields. The sum is again over all elementary plaquettes. The Hamiltonian is invariant under a $Z_{2}$ gauge transformation: $\mathbf{n}_{r} \rightarrow-\mathbf{n}_{r}, \sigma_{r r^{\prime}}=-\sigma_{r r^{\prime}}$ (all $r r^{\prime}$ links involving $r)$.

The Hamiltonian in Eq. 77 depends on two energies scales: $J$ as the microscopic nematic interaction and $K$ as the core energy of a $\pi$ spin disclination. At $K \rightarrow 0$, the $Z_{2}$ field is activated. As $J$ decreases, a spin nematic state is driven into an isotropic state where the free energy of an infinity long $\pi$ disclination is finite. The phase transition is of the first order and believed to be the one described by the Landau theory. At $K \rightarrow \infty$, the $Z_{2}$ gauge field is frozen. In this case, as $J$ decreases, the nematic state is driven into another isotropic state where the free energy of a $\pi$ disclination is proportional to the length of it. The transition is of the second order.

The gauge theory description of the classical nematic liquid predicts two isotropic and optically transparent liquids. They are different only in topological order. One of them has the hidden $Z_{2}$ order and the other one doesn't. A recent interesting experiment by Tokyo liquid crystal group did support this scenario 88. The optical turbidity measurement showed a transition between an optically turbid phase, which apparently can be identified as a nematic phase and an optically transparent state which represents an isotropic liquid. However, within the isotropic liquid, the dynamical slow down measurement indicates an additional phase transition between two optically transparent phases. Though it is not clear at the moment whether these two phases carry different topological order, the situation there is quite encouraging.

\section{Fermionic nematic liquids}

Finally, I want to remark on other electronic liquids which are believed to be "nematic" in nature. The first one is the ground state of $\mathrm{Sr}_{2} \mathrm{RuO}_{4}$, which was recently confirmed to be a p-wave superconducting state in a series of beautiful experiments $89,90,91,92$. In a p-wave superconductor of $\mathrm{Sr}_{2} \mathrm{RuO}_{4}$, a Cooper pair is a spin triplet and experiments further suggest that it be an Anderson-Morel state 61. If a pair is considered as a composited boson, then it occupies a $m_{F}=0$ channel of spin one states, up to a rotation of the quantization axis. The order parameter can be written in a tensor form as

$$
\Delta_{\alpha \beta}=\Delta_{0}(T) \exp (i \chi)\left(\sigma_{2} \sigma \cdot \mathbf{n}\right)_{\alpha \beta}\left(\mathbf{k}_{x} \pm i \mathbf{k}_{y}\right)
$$

in the absence of spin-orbit couplings, with $\sigma_{i}, i=0,1,2$ being Pauli matrices. By examining this pairing wave function, one expects that many aspects of $\mathrm{Sr}_{2} \mathrm{RuO}_{4}$ should be very similar to the spinor BEC described above. In particular, the concepts of the hidden symmetries and hidden gauge fields should be applicable in those systems [47]. Of course, in addition to this, there is a new twist in p-wave superconductors, that is the broken time invariance and broken parity 93. It leads to possible statistical transmutation of topological excitations and quasiparticles. This remains to be understood in the context of quantum nematic states discussed in this article. Results on spinor BECs discussed in this article will have no doubt to be demonstrative for the understanding of the physics in $\mathrm{Sr}_{2} \mathrm{RuO}_{4}$ superconducting crystals.

The Fermionic nematic liquids were also suggested for cuprates recently. The nematic model discussed in [73], and the QSNS in the spinor BEC discussed here are 
intimately connected with the recent $Z_{2}$ gauge theory developed for high- $T_{c}$ superconductors 46. To understand the connection between a "fractionalized" metal (non Fermi liquid) and a normal metal(Fermi liquid), the authors of that work pointed out that fractionalization and confining issues in cuprates can be partially understood by taking into account an inversion symmetry in a spinon-chargon model. In anisotropic phases of some strongly correlated electron systems such as "quantum Hall nematic phases", fermionic nematic liquids, or nematic metals were also investigated by Kivelson, Fradkin and their collaborators 94,95. V. Organnesyan et al. recently showed the breakdown of the fermi liquid theory in electronic nematic phases.

The investigations carried out for the spinor BEC therefore have possible impacts on the understanding of spin-charge separation or electron fractionalization phenomenon, which was first envisioned for cuprates about 15 years ago $96,97,98,99$. As one of the most interesting inventions by theorists in condensed matter physics, spincharge separation still continuously receives a lot of attentions from condensed matter physicists. Though microscopically BECs studied here are very different from those strongly correlated electronic systems, the long wave length physics do bear many similarities to some models employed for electron liquids. Studies of the quantum spin nematic states, in particular the quantum orders and spin fractionalization [44,45], not only are important for the understanding of spinor BECs, but also shed some light on some long standing issues in manybody physics. Being free of imperfections and easy to be manipulated magnetically or optically, the QSNS might be a potentially new platform for exploring some basic ideas in strongly correlated electron systems, which have not been settled.

\section{Acknowledgement}

It is my pleasure to thank F.A. Bais, G. 't Hooft, J. Ho, S. Kivelson, R. Moessner, M. Parikh, N. Read, P. Stamp, T. Senthil, J. Smit, B. Spivak, O. Starykn, D. Thouless, P. B. Wiegmann, B. de Wit for discussions. I am also grateful to E. Demler and D. F. Haldane for collaborations and many stimulating discussions, J. Chadi and N. Wingreen for their encouragement. This work was partially supported by ARO under DAAG 5598-01-0270 at Princeton University and a grant from NECRI, Princeton, USA. Finally, I want to thank the summer 2001 workshop of "Fundamental issues in quantum gases" at ASPEN center for Physics and Amsterdam summer workshop 2001 on "topology and statistics" for their hospitalities.

\section{APPENDIX A: SPIN WAVE FUNCTIONS IN $(U, V)$ REPRESENTATION}

In $(u, v)$ representation, states of spin $S$ are polynomials of degree $2 S$ in terms of $u, v$. Examples for $S=0,1,2$ are given below

$$
\begin{aligned}
& \mid S=0, S_{z}=0>=u^{0} \rightarrow 1 ; \\
& \mid S=\frac{1}{2}, S_{z}=\frac{1}{2}>=\sqrt{\frac{3}{2}} u \rightarrow \sqrt{\frac{3}{2}} \cos \frac{\theta}{2} \exp \left(i \frac{\phi}{2}\right), \\
& \mid S=\frac{1}{2}, S_{z}=-\frac{1}{2}>=\sqrt{\frac{3}{2}} v \rightarrow \sqrt{\frac{3}{2}} \sin \frac{\theta}{2} \exp \left(-i \frac{\phi}{2}\right) ; \\
& \mid S=1, S_{z}=1>=\sqrt{3} u^{2} \rightarrow \sqrt{3} \cos ^{2} \frac{\theta}{2} \exp (i \phi), \\
& \mid S=1, S_{z}=0>=\sqrt{6} u v \rightarrow \sqrt{6} \sin \theta \\
& \mid S=1, S_{z}=-1>=-\sqrt{3} v^{2} \rightarrow-\sqrt{3} \cos ^{2} \frac{\theta}{2} \exp (-i \phi) .
\end{aligned}
$$

Finally,

$$
\begin{aligned}
& \sqrt{6} u(\mathbf{n}) v(\mathbf{n})=\cos \theta \mid 1,0>+ \\
& \frac{1}{\sqrt{2}} \sin \theta \exp (-i \phi) \mid 1,1>+ \\
& \frac{-1}{\sqrt{2}} \sin \theta \exp (i \phi) \mid 1,-1>
\end{aligned}
$$

where $\mathbf{n}=(\theta, \phi)$. At $\theta=\pi / 2$, the amplitude of $\mid S=1, S_{z}=0>$ state vanishes.

\section{APPENDIX B: DERIVATION OF THE RG EQUATION}

Following [68], we introduce the unit vector $\mathbf{n}$ in a form of

$$
\begin{aligned}
& \mathbf{n}=\left(1-\sum_{\eta} \phi_{\eta}^{2}\right)^{1 / 2} \mathbf{e}_{0}+\sum_{\eta} \phi_{\eta} \mathbf{e}_{\eta} \\
& \mathbf{e}_{0} \cdot \mathbf{e}_{\eta}=0, \mathbf{e}_{\eta} \cdot \mathbf{e}_{\eta^{\prime}}=\delta_{\eta \eta^{\prime}}
\end{aligned}
$$

$\eta=1,2 . \phi_{\eta}$ can be considered as spin waves (fast variables) around given slowly varying $\mathbf{e}_{0}(x)$.

Given the constraints on $\mathbf{e}_{0}, \mathbf{e}_{\eta}$ in Eq. B1, we further introduce

$$
\partial_{\mu} e_{0}=B_{\mu}^{0 \eta} \mathbf{e}_{\eta}, \partial_{\mu} \mathbf{e}_{\eta}=A_{\mu}^{\eta \eta^{\prime}} \mathbf{e}_{\eta^{\prime}}-B_{\mu}^{0 \eta} \mathbf{e}_{0}
$$

Inserting Eqs.B1, B2 into Eq.13 and expanding (1 $\left.\sum_{\eta} \phi_{\eta}^{2}\right)$ in terms of $\phi_{\eta}$, one obtains

$$
\begin{aligned}
& \mathcal{L}_{s}=\frac{1}{2 f} \sum_{\eta}\left[B_{\mu}^{0 \eta}\right]^{2}+\frac{1}{2 f} \sum_{\eta}\left(\partial_{\mu} \phi_{\eta}+A_{\mu}^{\eta \eta^{\prime}} \phi_{\eta^{\prime}}\right)^{2} \\
& +\frac{1}{2 f} \sum_{\eta \eta^{\prime}} B_{\mu}^{0 \eta} B_{\mu}^{0 \eta^{\prime}}\left[\phi_{\eta} \phi_{\eta^{\prime}}-\delta_{\eta \eta^{\prime}} \sum_{\eta} \phi_{\eta}^{2}\right] .
\end{aligned}
$$


By integrating out fluctuations of $\phi$ from $e^{-l}<|\mathbf{p}|<1$ and $e^{-l}<|\epsilon|<1$ and restoring the momentum and the energy range, we obtain recursion relations for $f(l)$ in $d+1$ dimension

$$
\begin{aligned}
& \frac{1}{f(l)}=e^{l(d-1)}\left[\frac{1}{f(0)}-\Pi(l)\right], \\
& \Pi(l)=\int_{e^{-l}}^{1} \frac{d^{d} \mathbf{q}}{(2 \pi)^{d}} \frac{d \omega}{2 \pi} \frac{1}{\omega^{2}+\mathbf{q}^{2}} .
\end{aligned}
$$

The RG equation following Eq.B4 is

$$
\frac{d f}{d l}=(1-d) f+R_{d} f^{2},
$$

with $R_{1}=1 / 2 \pi, R_{2}=1 / 2 \pi^{2}, R_{3}=1 / 4 \pi^{2}$.

\section{APPENDIX C: CALCULATIONS OF SPREADS AT A ZERO FIELD LIMIT}

In spherical coordinates,

$$
\begin{aligned}
& \mathbf{L}_{x}=i \hbar\left(\sin \phi \frac{\partial}{\partial \theta}+\cot \theta \cos \phi \frac{\partial}{\partial \phi}\right) \\
& \mathbf{L}_{y}=i \hbar\left(-\cos \phi \frac{\partial}{\partial \theta}+\cot \theta \sin \phi \frac{\partial}{\partial \phi}\right) \\
& \mathbf{L}_{z}=i \hbar \frac{\partial}{\partial \phi}
\end{aligned}
$$

Taking into account Eq.C1, we obtain

$$
\begin{aligned}
& <\left(\delta^{2} \mathbf{n}\right)>_{t}=\frac{1}{B} \sum_{l}\left(1-M_{l, l^{\prime}}\right) \times \\
& \exp \left(-\frac{l^{2}}{4 \sigma}-i t \frac{c_{2} \rho l^{2}}{2 N}\right) \exp \left(-\frac{l^{\prime 2}}{4 \sigma}+i t \frac{c_{2} \rho l^{\prime 2}}{2 N}\right), \\
& M_{l^{\prime}, l}=\delta_{l^{\prime}, l}\left[\frac{(l+1)^{2}}{(2 l+1)(2 l+3)}+\frac{l^{2}}{(2 l+1)(2 l-1)}\right]+ \\
& \delta_{l^{\prime}, l+2}\left[\frac{(l+1)(l+2)}{(2 l+1)(2 l+3)}\right]+\delta_{l^{\prime}, l-2}\left[\frac{l(l-1)}{(2 l+1)(2 l-1)}\right] .
\end{aligned}
$$

In the limit $\sigma \gg 1$, we obtain results in Eq.74 with

$$
A_{0}=\frac{1}{B} \sum_{l} \frac{l^{2}}{4 \sigma} \exp \left(-\frac{l^{2}}{2 \sigma}\right) \text {. }
$$

\section{APPENDIX D: CALCULATIONS OF $<\delta^{2} \mathbf{N}>_{T}$ AT $H \neq 0$}

At $H \neq 0$, the equation of motion becomes particularly simple. $\mathbf{L} \rightarrow l_{\theta}=i \hbar \partial / \partial \theta$ and $\mathbf{n}=(\cos \theta, \sin \theta) ; \theta$ is the coordinate on a unit circle.
A wave packet of interest where $<\mathbf{n}_{x}>\sim 1,<\mathbf{n}_{y}>=$ 0 can be written as

$$
\Psi(\mathbf{n}, t)=\frac{1}{B} \sum_{l=0, \pm 2 \ldots} \exp \left(-\frac{l^{2}}{4 \sigma}\right) \exp \left(i l \theta-i t l^{2} \frac{c_{2} \rho}{2 N}\right)
$$

where $\sigma \gg 1$ is the spread of $l_{\theta}$ in the wave packet. One can again show that the energy, spin and the corresponding spread of $\mathbf{n}$ are

$$
\begin{aligned}
& \Delta E=\frac{2 \sigma c_{2} \rho}{N},<l_{\theta}^{2}>=4 \sigma A_{0} \\
& <\delta^{2} \mathbf{n}_{y}>_{t}=A_{0}\left[\frac{1}{\sigma}+4 \sigma\left(\frac{t c_{2} \rho}{N}\right)^{2}\right] .
\end{aligned}
$$

$A_{0}(\sigma)$ is a quantity of unity. The last equation in Eq.D2 is valid when $t<1 / \Delta E \sqrt{\sigma}$. In deriving Eq.D2, we employ Eq.C2 and notice that

$$
M_{l^{\prime}, l}=\delta_{l^{\prime}, l}+\frac{1}{2}\left[\delta_{l^{\prime}, l+2}+\delta_{l^{\prime}, l-2}\right]
$$

in this case.

[1] M. H. Anderson, J. R. Ensher, M. R. Matthews, C. E. Wieman, and E. A. Cornell, Science 269, 198(1995).

[2] K. B. Davis, M.O. Mewes, M. A. Joffe, M. R. Andrews, and W. Ketterle, Phys. Rev. Lett. 74, 5202(1995).

[3] C. C. Bradley, C.A. Sackett, J. J. Tollet, and R. G. Hulet, Phys. Rev. Lett. 75, 1687(1997).

[4] P. Nozieres and D. Pines, The theory of Quantum liquids (Addison-Wesley, Redwood City, CA 1990).

[5] Bose-Einstein condensation edited by A. Griffin, D. W. Snoke and S. Stringari (Cambridge University Press, Cambridge, 1995).

[6] F. Dalfovo, S. Giorgini, L. Pitaevskii and S. Stringari, Rev. Mod. Phys. vol 71, 463(1999).

[7] For a review, see W. Ketterle, D. S. Durfee, and D. M. Stamper-Kurn, in Bose-Einstein condensate in atomic gases, Proceedings of the International School of Physics Enrico Fermi, Course CXL, edited by M. Inguscio, $\mathrm{S}$ Stringari and C. E. Wieman (ISO Press, Amsterdam, 1999).

[8] W. C. Stwalley, Phys. Rev. Lett. 37, 1628 (1976).

[9] E. Tiesinga, B. J. Verhaar, and H. T. Stoof, Phys. Rev. A 47, 4114(1993).

[10] S. Inouye, M. R. Anderews, J. Stenger, H.J. Miesner, D. M. Stamper-Kurn, and W. Ketterle, Nature 392, 151(1998).

[11] Ph. Courteille, R. S. Freeland, and D. J. Heinzen, Phys. Rev. Lett. 81, 69(1998).

[12] J. Roberts, N. R. Claussen, James P. Burke, Jr., Chris H. Greene, E. A. Cornell, and C. E. Wieman, Phys. Rev. Lett. 81, 5109(1998). 
[13] V. Vuletic, A. J. Kerman, C. Chin, and S. Chu, Phys. Rev. Lett. 82, 1406(1999).

[14] S. L. Cornish, N. R. Claussen, J. L. Roberts, E. A. Cornell, and C. E. Wieman, cond-mat/0004290.

[15] C. A. Sackett, J. M. Gerton, M. Welling, and R. G. Hulet, Phys. Rev. Lett. 82, 876(1999)

[16] R. A. Duine and T. C. Stoof, cond-mat/0007026.

[17] M. R. Andrews, C. G. Townsend, H. J. Miesner, D. S. Durfee, D. M. Kurn, and W. Ketterle, Science 275, 637(1997).

[18] B.P. Anderson and M. A. Kasevich, Science 282, 1686(1998).

[19] M. R. Matthews, B. P. Anderson, P.C. Haljan, D. S. Hall, C. E. Wieman, and E. A. Cornell, Phys. Rev. Lett. 83, 2498(1999); See also J. E. Williams and M. J. Holland, Nature 401, 568(1999).

[20] K. W. Madison, F. Chevy, W. Wohlleben, and J. Dalibard, Phys. Rev. Lett. 84, 806(2000).

[21] F. Chevy, K. Madison, and J. Dalibard, condmat/0005221.

[22] C. Raman, M. Kohl, R. Onofrio, D. S. Durfee, C. E. Kuklewicz, Z. Hadzibabic, and W. Ketterle, Phys. Rev. Lett. 83, 2502(1999).

[23] O. M. Marago, S. A. Hopkins, J. Arlt, E. Hodby, G. Hechenblaikner, and C. J. Foot Phys. Rev. Lett. 84, 2056(2000).

[24] D. Guery-Odelin and S. Stringari, Phys. Rev. Lett. 83, 4452(1999).

[25] D.S.Hall, M. Matthews, C. Wieman, and E. Cornell, Phys. Rev. Lett.81, 1543(1998).

[26] D. M. Stamper-Kurn, H. J. Miesner, S. Inouye, M. Y. Andrews, and W. Ketterle, Phys. Rev. Lett 81, 500(1998).

[27] M. O. Mewes, M. R. Andrews, N. J. van Druten, D. M. Kurn, D. S. Durfee, C. G. Townsend, and W. Ketterle, Phys. Rev. Lett. 77, 988(1996).

[28] M. R. Andrews, D. M. Kurn, H. J. Miesner, D. S. Durfee, C. G. Townsend, S. Inouye, and W. Ketterle, Phys. Rev. Lett. 79, 553(1997).

[29] C. J. Myatt, E. A. Burt, R. W. Ghrist, E. A. Cornell, and C. E. Wieman, Phys. Rev. Lett. 78, 586(1997).

[30] D. M. Stamper-Kurn, M. R. Andrews, A. P. Chikkatur, S. Inouye, H. J. Miesner, J. Stenger, and W. Ketterle, Phys. Rev. Lett. 80, 2027(1998).

[31] J. Stenger, S. Inouye, D. M. Stamper-Kurn, H. J. Miesner, A. P. Chikkatur, and W. Ketterle, Nature 396, 345(1998).

[32] T. L. Ho, Phys. Rev. Lett. 81, 742 (1998).

[33] T. Ohmi and K. Machinda, J. Phys. Soc. Jpn. 67, 1822(1998).

[34] C. K. Law, H. Pu, and N. P. Bigelow, Phys. Rev. Lett. 81, 5257(1998).

[35] Y. Castin and C. Herzog, cond-mat/0012040.

[36] T.L. Ho and S. K. Yip, Phys. Rev. Lett. 84, 4031(2000).

[37] F. Zhou and F. D. Haldane, ITP-UU-00/51(2000).

[38] F. Zhou, Phys. Rev. Lett.87, 080401(2001).

[39] V. Leonhart and G. E. Volovik, JETP Lett.72, 46(2000).

[40] H. Stoof, E. Vliegen, and U. Al Khawaja, Phys. Rev. Lett. 87, 120407(2001).

[41] F. D. M. Haldane, Phys. Rev. Lett. 50, 1153(1983).

[42] E. Demler, F. Zhou, and F. D. Haldane, ITP-UU01/09(2001).

[43] E. Demler and F. Zhou, Phys. Rev. Lett.88, 163001(2002).

[44] Fei Zhou, cond-mat/0107263; ITP-UU-01/24(2001).
[45] Fei Zhou, cond-mat/0106133; ITP-UU-01/23(2001).

[46] T. Senthil and M. Fisher, cond-mat/9910224.

[47] E. Demler, C. Nayak, H. Y. Kee, Y. B. Kim and T. Senthil, cond-mat/0105446.

[48] D. P. Arovas, A. Auerbach and F. D. M. Haldane, Phys. Rev. Lett. 60, 531(1988).

[49] I. Afflect, J. Phys. Condensed Matter 1, 3047(1989).

[50] G. Grynberg, B. Lounis, P. Verkerk, J. Y. Courtois, and C. Salomon, Phys. Rev. Lett. 70, 2249 (1993).

[51] S.E. Hamann, D. L. Haycock, G. Klose, P. H. Pax, I. H. Deutsch, and P. S. Jessen, Phys. Rev. Lett 80, 4149 (1998)

[52] S. Friebel, C. D'Andrea, J. Walz, M. Weitz, and T.W. Hansch, Phys.Rev.A 57, R20 (1998)

[53] M. Rayzen, C. Salomon and Q. Niu, Phys. Today 50.

[54] L. Guidoni and P. Verkerk, Phys.Rev.Lett. 79, 3363 (1997).

[55] K.I. Petsas, A.B. Coates, and G. Grynberg, Phys.Rev.A 50, 5173 (1994).

[56] I.H. Deutsch and P.S. Jessen, Phys.Rev.A 57, 1972 (1998).

[57] D.J. Han, S. Wolf, S. Oliver, C. McCormick, M. T. DePue, and D. S. Weiss, Phys.Rev.Lett 85, 724 (2000).

[58] G. Toulouse and M. Kleman, Jour. Phys. Lett. (Paris) 37, L-149(76)

[59] G. Volovik and V. Mineev, Soviet Phys. JETP 45, 1186(77).

[60] D. N. Mermin, Rev. Mod. Phys. 51, 591(1979).

[61] See also D. Vollhardt and P. Wolfle, The superfluid phases of $\mathrm{He}^{3}$, Taylor and Francis, New York (1990). for discussions on topological defects in spin triplet fermionic superfluid phases.

[62] The vortex core is ferromagnetic with spins oriented along the direction of the flux, Haldane, unpublished.

[63] Fei Zhou, in preparation.

[64] J.Striet and S. Bais, hep-th/0108048.

[65] S. Blaha, Phys. Rev. Lett. 36, 874(1976).

[66] Y. S. Wu and A. Zee, Nucl. Phys. B324, 623(1989).

[67] P. W. Anderson, Phys. Rev. 56, 694(1952).

[68] A. M. Polyakov, Gauge Fields and Strings, Hardwood academic publishers (1987).

[69] S. Shankar and N. Read, Nucl. Phys. B 336, 457(1990).

[70] In a lattice model of $S^{2} / Z_{2}$ symmetry, $\pi$ spin disclination does not affect the gap, following Haldane(unpublished).

[71] P. G. de Gennes, The physics of liquid crystals, Oxford University Press (1974).

[72] F. Wegner, J. Math. Phys. 12, 2259(1971).

[73] P. Lammert, D. Rokhsar and J. Toner, Phys. Rev. Lett. 70, 1650(1993).

[74] S. Chakravarty and S. Kivelson, cond-mat/0012305.

[75] For a review, see P. W. Anderson, The theory of superconductivity in the High-T $T_{c}$ cuprates, Princeton University Press, Princeton(1997).

[76] This notion was introduced to me by P. W. Anderson in a series of lectures he gave at Princeton in 1999.

[77] E. Fradkin and S. H. Shenker, Phys. Rev. D 19, 3682(1979).

[78] X. G. Wen, cond-mat/0107071 and references therein. For early works on quantum orders in magnetic systems, see also V. Kalmeyer and R. B. Lauglin, Phy. Rev. 59, 2095(1987); P. B. Wiegmann, Phys. Rev. Lett.60, 821(1988); X. G. Wen, F. Wilczek and A. Zee, Phys. Rev. B 39, 11413(1989). 
[79] Additional

high derivative terms usually stablize Skyrmions at a finite $\xi$.

[80] This solution can be justified in the context of $C P^{N}$ fields, in a large- $N$ expansion. See A. M. Polyakov, Gauge Fields and Strings, Hardwood academic publishers (1987).

[81] A. Auerbach, B. Larson and G. N. Murthy, Phys. Rev. B 43, 11515(1991).

[82] G. N. Murthy and S. Sachdev, Nucl. Phys. B 344, 557(1990).

[83] $\mathrm{N}$. Read, private communication.

[84] For an excellent review, see A. J. Heeger, S. Kivelson, W. P. Su and J. R. Schrieffer, Rev. Mod. Phys. 60, 781(1988).

[85] J. Villain, J. Phys. (Paris), 38, 26(1977); J. Villain et al., J. Phys. (Paris), 41, 1263(1980).

[86] E. F. Shender, Sov. Phys. JETP, 56, 178(1982).

[87] C. L. Henley, Phys. Rev. Lett. 62, 2056(1989).

[88] J. Yamamoto and H. Tanaka, Nature 409, 321(2001).

[89] Y. Maeno et al., Nature 372, 532(1994).

[90] A. P. Mackenzie, S. R. Julian, A. J. Diver, G. J. McMullan,
M. P. Ray, G. G. Lonzarich, Y. Maeno, S. Nishizaki, and T. Fujita, Phys. Rev. Let. 76, 3786(1996).

[91] K. Ishida et al., Nature 396, 658(1998).

[92] J. A. Duffy, S.M. Hayden, Y. Maeno, Z. Mao, J. Kulda, and G. I. McIntyre, Phys. Rev. Lett. 85, 5412(200).

[93] G. E. Volovik and V. M. Yakovenko, J. Phys. C 1, 5263(1989).

[94] E. Fradkin, S. Kivelson, E. Manousakis, and K. Nho, Phys. Rev. Lett. 84, 1982(2000).

[95] V. Oganesyan, S. Kivelson, and E. Fradkin, conmat/0102093

[96] P. W. Anderson, Science 235, 1196(1987).

[97] G. Baskaran, Z. Zou and P. W. Anderson, Solid Sate Commun. 63, 873(1987).

[98] S. A. Kivelson, D. Rokhsar, and J.P. Sethna, Phys. Rev. B 35, 8865(1987); D.S. Rokhsar and S.A. Kivelson, Phys. Rev. Lett. 61, 2376(1988). Also R. Moessner and S. L. Sondhi, Phys. Rev. Lett. 86, 1881(2001).

[99] P. B. Wiegmann, Phys. Rev. Lett. 60, 821(1988). 